\title{
THE CONSISTENCY STRENGTH OF LONG PROJECTIVE DETERMINACY
}

\author{
JUAN P. AGUILERA AND SANDRA MÜLLER
}

\begin{abstract}
We determine the consistency strength of determinacy for projective games of length $\omega^{2}$. Our main theorem is that $\boldsymbol{\Pi}_{n+1}^{1}$-determinacy for games of length $\omega^{2}$ implies the existence of a model of set theory with $\omega+n$ Woodin cardinals. In a first step, we show that this hypothesis implies that there is a countable set of reals $A$ such that $M_{n}(A)$, the canonical inner model for $n$ Woodin cardinals constructed over $A$, satisfies $A=\mathbb{R}$ and the Axiom of Determinacy. Then we argue how to obtain a model with $\omega+n$ Woodin cardinal from this.

We also show how the proof can be adapted to investigate the consistency strength of determinacy for games of length $\omega^{2}$ with payoff in $\partial^{\mathbb{R}} \boldsymbol{\Pi}_{1}^{1}$ or with $\sigma$-projective payoff.
\end{abstract}

$\S 1$. Introduction. We study the consistency strength of determinacy for games of length $\omega^{2}$ with payoff in various pointclasses $\Gamma$. Specifically, given a set $A \subset \omega^{\omega^{2}}$, i.e., a set of sequences of natural numbers of length $\omega^{2}$, with $A \in \Gamma$, consider the following game:

$$
\begin{array}{l|lllll}
\mathrm{I} & n_{0} & n_{2} & \ldots & n_{\omega} & \ldots \\
\hline \mathrm{II} & n_{1} & n_{3} & \ldots & n_{\omega+1} & \ldots
\end{array}
$$

Players I and II alternate turns playing natural numbers to produce some $x=$ $\left(n_{0}, n_{1}, \ldots, n_{\omega}, n_{\omega+1}, \ldots\right) \in \omega^{\omega^{2}}$. Player I wins such a run $x$ of the game if, and only if, $x \in A$; otherwise, Player II wins. We study the strength of the statement that games of this form are determined, i.e., that one of the players has a winning strategy. For all nontrivial classes $\Gamma$, this question is independent of Zermelo-Fraenkel set theory with the Axiom of Choice (ZFC); for some of them, however, it is known to follow from natural strengthenings of ZFC, namely, from assumptions on the existence of large cardinals.

Recall that the projective subsets of a Polish space are those obtainable from Borel sets in finitely many stages by applying complements and projections from a finite power of the space. We are mainly interested in the case where $\Gamma$ is a projective pointclass, i.e., $\boldsymbol{\Pi}_{n}^{1}$ for some natural number $n$, but we will also consider the cases in which $\Gamma$ is equal to $\partial^{\mathbb{R}} \boldsymbol{\Pi}_{1}^{1}$ or a $\sigma$-projective pointclass. ${ }^{1}$

Received January 25, 2019.

2010 Mathematics Subject Classification. 03E45, 03E60, 03E15, 03 E55.

Key words and phrases. infinite game, determinacy, inner model theory, large cardinal, long game, mouse.

${ }^{1}$ The pointclass of all $\sigma$-projective sets is the smallest pointclass closed under complements, countable unions, and projections, where countable unions refer to sets which are subsets of the same product space. Moreover, we as usual identify $\mathbb{R}$ and ${ }^{\omega} \omega$.

(C) 2019, Association for Symbolic Logic 0022-4812/20/8501-0016 DOI: $10.1017 /$ js 1.2019 .78 
The study of games of length $\omega^{2}$ is motivated by the folklore result that projective determinacy for games of length $\omega$ implies projective determinacy for games of length $\alpha$, for any $\alpha<\omega^{2}$. It is also not difficult to see that $\Pi_{n+1}^{1}$-determinacy of length $\omega^{2}$ follows from analytic determinacy for games of length $\omega \cdot(\omega+n)$, for natural numbers $n .^{2}$

Analytic determinacy for games of length $\omega \cdot(\omega+n)$ was proved by Neeman [23, Theorem 2A.3] from a large cardinal hypothesis. Specifically, he assumed the existence of a weakly iterable model of set theory with $\omega+n$ Woodin cardinals, along with a sharp for the model. In fact, he proved this result for analytic games of any fixed countable length $\omega \cdot(\theta+1)$, from corresponding assumptions. This naturally yields the question whether his results are optimal, i.e., whether the determinacy of these long games implies the existence of models with certain numbers of Woodin cardinals. In light of this, we prove the following theorem:

THEOREM 1.1. Suppose that $\boldsymbol{\Pi}_{n+1}^{1}$-determinacy for games of length $\omega^{2}$ holds and let $x \in{ }^{\omega} \omega$ be arbitrary. Then, there is a proper class model M of ZFC with $\omega+n$ Woodin cardinals such that $x \in M$.

The Woodin cardinals of the model we construct in the proof of Theorem 1.1 are in reality countable. Moreover, the model is a premouse, i.e., fine structural, and we can in fact construct it such that it is active, i.e., it has a sharp on top. As a corollary of the theorem and the results from [23], we obtain the following equiconsistencies, which also connect projective determinacy for games of length $\omega^{2}$ to projective determinacy for games on reals. ${ }^{3}$

COROLLARY 1.2. The following schemata are equiconsistent:

(1) ZFC $+\left\{\right.$ " $\Pi_{n}^{1}$-determinacy for games of length $\omega^{2}$ on $\omega$ " : $\left.n \in \omega\right\}$.

(2) $\mathrm{ZF}+\mathrm{DC}+\mathrm{AD}+\{$ "there are $n$ Woodin cardinals": $n \in \omega\}$.

(3) ZFC $+\{$ "there are $\omega+n$ Woodin cardinals": $n \in \omega\}$.

(4) $\mathrm{ZF}+\mathrm{DC}+\mathrm{AD}+\left\{\right.$ “ $\boldsymbol{\Pi}_{n}^{1}$-determinacy for games of length $\omega$ on $\mathbb{R}$ " $\left.: n \in \omega\right\}$.

(5) $\mathrm{ZF}+\mathrm{DC}+\mathrm{AD}+\left\{“ V^{\operatorname{Col}(\omega, \mathbb{R})} \vDash \boldsymbol{\Pi}_{n}^{1}\right.$-determinacy for games of length $\omega$ on $\omega ”: n \in \omega\}$.

The proof of Theorem 1.1 has two main parts: in the first one, the hypothesis is shown to imply that for a closed and unbounded set of countable sets of reals $A$, there is a fine structural model of the Axiom of Determinacy with $n$ Woodin cardinals whose set of real numbers is precisely $A$. In the second part we use a Prikry-like partial order to force over these models and obtain via a translation procedure an infinite sequence of Woodin cardinals below the already existing ones. The proof of Corollary 1.2 is sketched in the final section of this article.

Background. The situation for games of length $\omega$ is well understood: There is a tight connection beween determinacy for these games and the existence of inner models with large cardinals. Martin [13] showed that Borel games are determined in ZFC. Contrary to that, determinacy for $\Sigma_{1}^{1}$ (i.e., analytic) games cannot be proved in ZFC

\footnotetext{
${ }^{2}$ In fact, an argument as in [3, Proposition 2.7] with a more careful analysis of the complexity of the payoff sets (using projective determinacy) shows that these two determinacy hypotheses are equivalent.

${ }^{3}$ We would like to thank the referee for asking whether (1.2) and (1.2) could be added to Corollary 1.2 ; see also [2].
} 
alone. By theorems of Martin [12] and Harrington [6] $\Sigma_{1}^{1}$ games are determined if, and only if, $x^{\sharp}$ exists for every $x \in{ }^{\omega} \omega$. Martin and Steel [14] proved, for each $n \in \omega$, that the existence of $n$ Woodin cardinals with a measurable cardinal above implies the determinacy of all $\Sigma_{n+1}^{1}$ sets. Woodin (unpublished) improved this for odd $n$ by showing that the existence of $M_{n}^{\sharp}(x)$, the canonical active $\omega_{1}$-iterable inner model with $n$ Woodin cardinals constructed over $x$, for all reals $x$ suffices to show $\Sigma_{n+1}^{1}$ determinacy. Afterwards, Neeman improved this in [22] even further and showed that for all $n$, the existence of $M_{n}^{\sharp}(x)$ for all reals $x$ implies determinacy of all $\partial^{n}\left(<\omega^{2}-\boldsymbol{\Pi}_{1}^{1}\right)$ sets. Concerning the other direction, Woodin (see [21]) showed that if $\Sigma_{n+1}^{1}$ games are determined, then $M_{n}^{\sharp}(x)$ exists for all reals $x$, thus establishing a level-by-level characterization of projective determinacy in terms of the existence of inner models with large cardinals. Similar characterizations are known for $\sigma$ projective games of length $\omega$ (see [1] and [3]). Determinacy for games of length $\omega$ with payoff in $\supset^{\mathbb{R}} \boldsymbol{\Pi}_{1}^{1}$ is equivalent to $\mathrm{AD}^{L(\mathbb{R})}$ (see [16]).

Woodin showed that the existence of $\omega^{2}$ Woodin cardinals under choice is equiconsistent with $\mathrm{AD}^{+}+\mathrm{DC}$ and the existence of a normal fine measure on $\mathcal{P}_{\omega_{1}}(\mathbb{R})$ (see Remark 9.98 in [42]). By this and a result of Trang (see [38, Theorem 2.3.11], case $\alpha=2$ ), determinacy of analytic games of length $\omega^{3}$ implies the consistency of $\omega^{2}$ Woodin cardinals under choice. In fact, a similar result holds for $\omega^{\alpha}$ Woodin cardinals and determinacy of analytic games of length $\omega^{1+\alpha}$ for all $1<\alpha<\omega$ and all limit ordinals $\omega \leq \alpha<\omega_{1}$. For details see Theorems 2.2.1 and 2.3.11 in [38], as well as [39] and [40].

Further results. The proof of Theorem 1.1 uses game arguments based on techniques from Martin and Steel [16] and [21], tracing back to arguments of H. Friedman [4] and and Solovay [11] as well as inner model theoretic methods based on the unpublished notes [31]. The method of proof of Theorem 1.1 can also be used to show the following two generalizations:

THEOREM 1.3. Suppose that $\supset^{\mathbb{R}} \boldsymbol{\Pi}_{1}^{1}$-determinacy for games of length $\omega^{2}$ holds and let $x \in{ }^{\omega} \omega$ be arbitrary. Then, there is a proper class model $M$ of ZFC with $\omega+\omega$ Woodin cardinals such that $x \in M$.

As above, it is not hard to show that determinacy of analytic games of length $\omega \cdot(\omega+\omega)$ implies determinacy of $\supset^{\mathbb{R}} \boldsymbol{\Pi}_{1}^{1}$ games of length $\omega^{2}$. Our methods also generalize to games with $\sigma$-projective payoff and premice of class $S_{\alpha}$ (see [1] and [3, Definition 4.1]).

THEOREM 1.4. Suppose that $\sigma$-projective determinacy for games of length $\omega^{2}$ holds and let $x \in{ }^{\omega} \omega$ be arbitrary. Then, for every $\alpha<\omega_{1}$, there is a proper class model $M$ of ZFC with $\omega$ Woodin cardinals with supremum $\lambda$ which is of class $S_{\alpha}$ above $\lambda$ and such that $x \in M$.

Outline. In Section 2, we establish conventions and recall some known facts about extender models which will be used later on. Focusing first on the case $n=1$, Section 3 contains the main argument and shows that determinacy for games of length $\omega^{2}$ with $\Pi_{2}^{1}$ payoff implies the existence of fine structural models of the Axiom of Determinacy with one Woodin cardinal. In Section 4, we argue that this fine structural model satisfies $\mathrm{DC}$ and $\mathrm{AD}^{+}$. In Section 5, we show how to obtain a model of ZFC with $\omega+1$ Woodin cardinals from this model. Finally, in Section 6 
we sketch the proof of Corollary 1.2 and explain how to carry out the modifications needed to prove Theorems 1.3 and 1.4.

We would like to thank John Steel for valuable discussions related to this article, in particular to Lemma 3.8, and for making the unpublished notes [31] available to us. Moreover, we would like to thank Grigor Sargsyan for his helpful comments on an earlier version of this manuscript. Finally, we would like to thank the referee for carefully reading our article and making several valuable suggestions.

§2. Preliminaries. For basic set theoretic definitions and results we refer to [8] and [18]. Moreover, we work with canonical, fine structural models with large cardinals, called premice. We refer the reader to, e.g., [35] for an introduction, and to [17], [28], and [33] for additional background. In particular, we will use Mitchell-Steel indexing for extender sequences and the notation from [35]. As in [33] we will consider relativized premice constructed over arbitrary transitive sets $X$. Let $\mathcal{L}_{\mathrm{pm}}=\{\dot{\epsilon}, \dot{E}, \dot{F}, \dot{X}\}$ denote the language of relativized premice, where $\dot{E}$ is the predicate for the extender sequence, $\dot{F}$ is the predicate for the top extender, and $\dot{X}$ is the predicate for the set over which we construct the premouse.

For a transitive set $X$, we say an $X$-premouse $M=\left(J_{\alpha}^{\vec{E}}, \in, \vec{E}, E_{\alpha}, X\right)$ for $\vec{E}=(\dot{E})^{M}, E_{\alpha}=(\dot{F})^{M}$, and $X=(\dot{X})^{M}$ is active if $E_{\alpha} \neq \emptyset$. Otherwise, we say $M$ is passive. We let $M \mid \gamma=\left(J_{\gamma}^{\vec{E}}, \in, \vec{E} \uparrow \gamma, E_{\gamma}, X\right)$ for $\gamma \leq M \cap$ Ord. Moreover, we write $M \| \gamma$ for the passive initial segment of $M$ of height $\gamma$, i.e., $M \| \gamma=\left(J_{\gamma}^{\vec{E}}, \in, \vec{E}\lceil\gamma, \emptyset, X)\right.$, for $\gamma \leq M \cap$ Ord. In particular, $M \| \operatorname{Ord}^{M}$ denotes the premouse $N$ which agrees with $M$ except that we let $(\dot{F})^{N}=\emptyset$. We say an ordinal $\eta$ is a (strong) cutpoint of $M$ if there is no extender $E$ on the $M$-sequence with $\operatorname{CRIT}(E) \leq \eta \leq \operatorname{lh}(E)$.

An arbitrary $X$-premouse might not satisfy the Axiom of Choice, but it can be construed as an ordinary premouse which satisfies the Axiom of Choice if a well order of $X$ is added generically. In particular, every $X$-premouse $M$ is well-ordered $\bmod X$, i.e., for every set $Y \in M$ there is an ordinal $\alpha$ and a surjection $h$ in $M$ such that $h: X \times \alpha \rightarrow Y$. In this article we shall mainly be interested in $X$-premice which satisfy the Axiom of Determinacy (and hence not the full Axiom of Choice), where $X \in \mathcal{P}_{\omega_{1}}(\mathbb{R})$, i.e., $X$ is a countable set of reals.

Throughout this article, we work under the assumption that all premice are tame, i.e., that there is no extender on the sequence of a premouse overlapping a Woodin cardinal. This results in no loss of generality, as otherwise the conclusions of the theorems in Section 1 hold.

In the rest of this section we summarize some facts about premice which we are going to need later. Most of these can be found in [21] and [41] for $x$-premice for a real $x$ and they straightforwardly generalize to $X$-premice for arbitrary countable sets of reals $X$. Fix such a set $X \in \mathcal{P}_{\omega_{1}}(\mathbb{R})$ for the rest of this section.

Definition 2.1. Let $M$ be an $X$-premouse and let $\alpha<\omega_{1}^{L}$. Then we say that $M$ is $\alpha$-small if, and only if, for every ordinal $\kappa \leq M \cap$ Ord such that there is an extender with critical point $\kappa$ on the $M$-sequence, in $M \mid \kappa$ there is no set of ordinals $W$ of order-type $\alpha$ such that every ordinal in $W$ is a Woodin cardinal in $M \mid \kappa$. 
If it exists and is unique, we denote the $\omega_{1}$-iterable, countable, sound $X$-premouse which is not $\alpha$-small, but all of whose proper initial segments are $\alpha$-small, by $M_{\alpha}^{\sharp}(X)$. In this case $M_{\alpha}(X)$ denotes the result of iterating the topmost measure of $M_{\alpha}^{\sharp}(X)$ and its images out of the universe.

We will now argue that under certain conditions there is a comparison lemma for $n$-small $X$-premice. This will be used in the proof of Theorem 3.1. For premice over a real $x$ this can be found, for example, in [21, Lemma 2.11]; the argument there generalizes to premice over countable sets of reals. For the reader's convenience we will briefly sketch the main ideas and recall the statements. The following notion will be important in what follows to ensure that $\mathcal{Q}$-structures exist.

Definition 2.2. Let $M$ be a sound $X$-premouse and let $\delta$ be a cardinal in $M$ or $\delta=M \cap$ Ord. We say that $\delta$ is not definably Woodin over $M$ if, and only if, there exists an ordinal $\gamma \leq M \cap$ Ord such that $\gamma \geq \delta$ and either

(i) over $M \mid \gamma$ there exists an $r \Sigma_{n}$-definable set $A \subset \delta$ for some $n<\omega$ such that for no $\kappa<\delta$ do the extenders on the $M$-sequence witness that $\kappa$ is strong up to $\delta$ with respect to $A$, or

(ii) $\rho_{n}(M \mid \gamma)<\delta$ for some $n<\omega$.

For several iterability arguments to follow we need our premice to satisfy the following property, which, as a fine structural argument shows, is preserved during iterations. This in turn ensures that $\mathcal{Q}$-structures exist in iterations of a premouse $M$ satisfying it.

Definition 2.3. Let $M$ be a sound $X$-premouse. We say $M$ has no definable Woodin cardinals if, and only if, for all $\delta \leq M \cap$ Ord we have that $\delta$ is not definably Woodin over $M$.

The proof of [21, Lemma 2.11] generalizes to $X$-premice and shows the following lemma. Here the hypothesis that $M_{n}^{\sharp}(x)$ exists for all $x \in \mathbb{R}$ is used to compare the countable premice $M$ and $N$ inside the model $M_{n}^{\sharp}(x)$ for a real $x$ coding $M$ and $N$. The fact that the iteration strategies for $M$ and $N$ are guided by $\mathcal{Q}$-structures ensures that their restriction to trees in $H_{\delta_{x}}^{M_{n}^{\sharp}(x)}$, where $\delta_{x}$ is the least Woodin cardinal in $M_{n}^{\sharp}(x)$, is in $M_{n}^{\sharp}(x)$ and hence $\omega_{1}^{V}$-iterability suffices for comparison in this situation.

Lemma 2.4. Suppose that $M_{n}^{\sharp}(x)$ exists for all $x \in \mathbb{R}$. Let $M$ and $N$ be countable $\omega_{1}$-iterable sound $X$-premice such that every proper initial segment of $M$ and $N$ is $n$-small and they both do not have definable Woodin cardinals. Then there are iterates $M^{*}$ and $N^{*}$ of $M$ and $N$, respectively, such that one of the following holds:

1. $M^{*}$ is an initial segment of $N^{*}$ and there is no drop on the main branch in the iteration from $M$ to $M^{*}$,

2. $N^{*}$ is an initial segment of $M^{*}$ and there is no drop on the main branch in the iteration from $N$ to $N^{*}$.

In the statement of Lemma 2.4 the assumption that $M$ and $N$ do not have definable Woodin cardinals can be replaced by the assumption that $M$ and $N$ do not have Woodin cardinals; see the remark after the proof of Lemma 2.11 in [21]. 
There is a variant of this lemma for the case that one of the premice is only $\Pi_{n}^{1}$-iterable as introduced in Definitions 1.4 and 1.6 in [30]. In this case, the last model of the iteration tree on that premouse need not be fully well founded, but the argument from [30, Lemma 2.2] (see also Corollary 2.15 in [21]) yields that we still have a comparison lemma.

Lemma 2.5. Suppose that $M_{n}^{\sharp}(x)$ exists for all $x \in \mathbb{R}$. Let $M$ and $N$ be countable $n$-small sound solid $X$-premice which both do not have Woodin cardinals. Moreover, assume that $M$ is $\omega_{1}$-iterable and $N$ is $\Pi_{n+1}^{1}$-iterable. Then there is an iteration tree $\mathcal{T}$ on $M$ and a putative 4 iteration tree $\mathcal{U}$ on $N$ of length $\lambda+1$ for some limit ordinal $\lambda$ such that one of the following holds:

1. $\mathcal{M}_{\lambda}^{\mathcal{T}}$ is an initial segment of $\mathcal{M}_{\lambda}^{\mathcal{U}}$ and there is no drop on the main branch through $\mathcal{T}$. In this case $\mathcal{M}_{\lambda}^{\mathcal{U}}$ need not be fully well founded, but it is well founded up to $\mathcal{M}_{\lambda}^{\mathcal{T}} \cap$ Ord.

2. $\mathcal{M}_{\lambda}^{\mathcal{U}}$ is an initial segment of $\mathcal{M}_{\lambda}^{\mathcal{T}}$ and there is no drop on the main branch through $\mathcal{U}$. In this case $\mathcal{M}_{\lambda}^{\mathcal{U}}$ is fully well founded and $\mathcal{U}$ is an iteration tree.

Finally, note that by a standard argument $\boldsymbol{\Pi}_{2}^{1}$-determinacy, or equivalently that $M_{1}^{\sharp}(x)$ exists and is $\omega_{1}$-iterable for all $x \in \mathbb{R}$, implies that $M_{1}^{\sharp}(X)$ exists and is $\omega_{1}$-iterable for any countable set of reals $X$.

§3. Models of the Axiom of Determinacy with a Woodin cardinal. Recall that $\mathcal{P}_{\omega_{1}}(\mathbb{R})$ denotes the set of all countable sets of reals. We consider models of the form $M_{n}(A)$, where $n \in \omega$ and $A \in \mathcal{P}_{\omega_{1}}(\mathbb{R})$. It is not hard to see that-provided they exist - many of these structures are models of the Axiom of Choice. Our first theorem shows that if games of length $\omega^{2}$ with $\boldsymbol{\Pi}_{n+1}^{1}$ payoff are determined, then many of these structures are models of the Axiom of Determinacy and we can in addition have that $M_{n}(A) \cap \mathbb{R}=A$.

THEOREM 3.1. Let $n \in \omega$ and suppose that determinacy for $\boldsymbol{\Pi}_{n+1}^{1}$ games of length $\omega^{2}$ holds. Then, there is a club $\mathcal{C} \subset \mathcal{P}_{\omega_{1}}(\mathbb{R})$ such that for all $A \in \mathcal{C}, M_{n}^{\sharp}(A)$ exists, is $\omega_{1}$-iterable, $M_{n}^{\sharp}(A) \cap \mathbb{R}=A$, and

$$
M_{n}(A) \models \mathrm{ZF}+\mathrm{AD} .
$$

To simplify the notation we will from this point on only consider the case $n=1$. The general case $n \in \omega$ can be shown by straightforward modifications of the proof we give for $n=1$ below. For the rest of this section assume that $V$ is a model of ZFC and Projective Determinacy, i.e., that $M_{n}^{\sharp}(x)$ exists for all reals $x$. Note that the hypothesis of Theorem 1.1, determinacy for $\boldsymbol{\Pi}_{n+1}^{1}$ games of length $\omega^{2}$, trivially implies Projective Determinacy since after the first $\omega$ moves the following rounds can be used to "play witnesses for projections." Whenever we are assuming a stronger determinacy hypothesis, we will point it out explicitly.

Before moving on to the proof of Theorem 3.1, we recall some basic modeltheoretic facts we will need later. In addition to the language of premice, $\mathcal{L}_{\mathrm{pm}}=$ $\{\dot{\epsilon}, \dot{E}, \dot{F}, \dot{X}\}$, we will now also consider the language $\mathcal{L}_{\mathrm{pm}}\left(\left\{\dot{x}_{i}: i \in \omega\right\}\right)$ resulting from enhancing $\mathcal{L}_{\mathrm{pm}}$ with constants $\dot{x}_{i}$, for $i \in \omega$. For an $\mathcal{L}_{\mathrm{pm}}\left(\left\{\dot{x}_{i}: i \in \omega\right\}\right)$-model

\footnotetext{
${ }^{4}$ We say that $\mathcal{U}$ is a putative iteration tree if it satisfies all properties of an iteration tree, but we allow the last model, if it exists, to be ill-founded.
} 
$\mathcal{M}=\left(M, \in, \dot{E}^{\mathcal{M}}, \dot{F}^{\mathcal{M}}, \dot{X}^{\mathcal{M}},\left\{\dot{x}_{i}^{\mathcal{M}}: i<\omega\right\}\right)$ we write $\mathcal{M} \uparrow \mathcal{L}_{\mathrm{pm}}$ for the restriction $\left(M, \in, \dot{E}^{\mathcal{M}}, \dot{F}^{\mathcal{M}}, \dot{X}^{\mathcal{M}}\right)$ of the model $\mathcal{M}$ to the smaller language $\mathcal{L}_{\mathrm{pm}}$.

Let $\mathcal{M}=\left(M, \in, \dot{E}^{\mathcal{M}}, \dot{F}^{\mathcal{M}}, \dot{X}^{\mathcal{M}},\left\{x_{i}: i \in \omega\right\}\right)$ for $x_{i}=\dot{x}_{i}^{\mathcal{M}}, i \in \omega$, be a model in the enhanced language, so in particular $\left\{x_{i}: i \in \omega\right\} \subseteq M$. The definable closure of $\left\{x_{i}: i \in \omega\right\}$ in $\mathcal{M} \uparrow \mathcal{L}_{\text {pm }}$ is defined to be the submodel

$$
\left(\bar{M}, \in, \dot{E}^{\mathcal{M}} \cap \bar{M}, \dot{F}^{\mathcal{M}} \cap \bar{M}, \dot{X}^{\mathcal{M}} \cap \bar{M}\right)
$$

of $\mathcal{M} \uparrow \mathcal{L}_{\text {pm }}$ where $\bar{M}$ consists of all $a \in M$ such that for some $k<\omega$ and some $\mathcal{L}_{\text {pm }}$-formula $\phi\left(v, v_{0}, \ldots, v_{k}\right)$,

$\mathcal{M}\left\lceil\mathcal{L}_{\mathrm{pm}} \models " \phi\left[a, x_{0}, \ldots, x_{k}\right]\right.$ and there is a unique $x$ such that $\phi\left[x, x_{0}, \ldots, x_{k}\right] . "$

For sufficiently nice theories $T$, the definable closure of a model of $T$ does not depend on the model itself but only on the theory.

Lemma 3.2. Suppose $T \supset$ ZF is a complete, consistent theory in the language $\mathcal{L}_{\mathrm{pm}}\left(\left\{\dot{x}_{i}: i \in \omega\right\}\right)$ with the property that whenever

$$
\mathcal{M}=\left(M, \in, \dot{E}^{\mathcal{M}}, \dot{F}^{\mathcal{M}}, \dot{X}^{\mathcal{M}},\left\{\dot{x}_{i}^{\mathcal{M}}: i \in \omega\right\}\right)
$$

is a model of $T$ and $\mathcal{N}^{\mathcal{M}}$ is the definable closure of $\left\{\dot{x}_{i}^{\mathcal{M}}: i \in \omega\right\}$ in $\mathcal{M} \uparrow \mathcal{L}_{\mathrm{pm}}$, then

(1) for each $i \in \omega, \mathcal{M} \models \dot{x}_{i}^{\mathcal{M}} \in \mathbb{R}$,

(2) $\mathcal{N}^{\mathcal{M}} \prec \mathcal{M} \uparrow \mathcal{L}_{\mathrm{pm}}$.

Then $\mathcal{N}^{\mathcal{M}}$ does not depend on $\mathcal{M}$, i.e., if $\mathcal{P}$ is another model of $T$ with properties (1) and (2), then $\mathcal{N}^{\mathcal{M}}$ and $\mathcal{N}^{\mathcal{P}}$ are isomorphic.

Proof. Let $T$ be as in the statement and let

$$
\mathcal{M}=\left(M, \in, \dot{E}^{\mathcal{M}}, \dot{F}^{\mathcal{M}}, \dot{X}^{\mathcal{M}},\left\{\dot{x}_{i}^{\mathcal{M}}: i \in \omega\right\}\right)
$$

and

$$
\mathcal{P}=\left(P, \in, \dot{E}^{\mathcal{P}}, \dot{F}^{\mathcal{P}}, \dot{X}^{\mathcal{P}},\left\{\dot{x}_{i}^{\mathcal{P}}: i \in \omega\right\}\right)
$$

be two models of $T$. Since $T$ is complete, $\mathcal{M}$ and $\mathcal{P}$ are elementarily equivalent with respect to the language $\mathcal{L}_{\mathrm{pm}}\left(\left\{\dot{x}_{i}: i \in \omega\right\}\right)$. If $\mathcal{N}^{\mathcal{M}}$ and $\mathcal{N}^{\mathcal{P}}$ are the respective definable closures of $\mathcal{M} \uparrow \mathcal{L}_{\mathrm{pm}}$ and $\mathcal{P}\left\lceil\mathcal{L}_{\mathrm{pm}}\right.$, the natural function $\rho$ given by

$$
\begin{aligned}
& \text { the unique } a \in M \text { such that } \mathcal{M} \uparrow \mathcal{L}_{\mathrm{pm}} \models \phi\left[a, \dot{x}_{1}^{\mathcal{M}}, \ldots, \dot{x}_{k}^{\mathcal{M}}\right] \\
& \mapsto \text { the unique } b \in P \text { such that } \mathcal{P} \uparrow \mathcal{L}_{\mathrm{pm}} \models \phi\left[b, \dot{x}_{1}^{\mathcal{P}}, \ldots, \dot{x}_{k}^{\mathcal{P}}\right]
\end{aligned}
$$

for some $k<\omega$ and some $\mathcal{L}_{\mathrm{pm}}$-formula $\phi\left(x, v_{1}, \ldots, v_{k}\right)$ is an isomorphism from $\mathcal{N}^{\mathcal{M}}$ to $\mathcal{N}^{\mathcal{P}}$. This follows from the following observations:

1. Since $\dot{x}_{i}$ are constants interpreted by reals and $T$ is complete, they have the same interpretation in $\mathcal{M}$ and $\mathcal{P}$, i.e., for all $i \in \omega, \dot{x}_{i}^{\mathcal{M}}=\dot{x}_{i}^{\mathcal{P}}$.

2. If $x \in \mathcal{N}^{\mathcal{M}}$, then there is an $\mathcal{L}_{\text {pm }}$-formula $\psi\left(v, v_{1}, \ldots, v_{k}\right)$ such that $\mathcal{M}$ $\mathcal{L}_{\mathrm{pm}} \models \psi\left[x, \dot{x}_{1}^{\mathcal{M}}, \ldots, \dot{x}_{k}^{\mathcal{M}}\right]$ and $x$ is unique with this property in $\mathcal{M} \uparrow \mathcal{L}_{\mathrm{pm}}$. Thus, if $x \in \dot{E}^{\mathcal{M}}$,

$$
\mathcal{M} \models \text { " } x \text { is the unique element satisfying } \psi\left[x, \dot{x}_{1}^{\mathcal{M}}, \ldots, \dot{x}_{k}^{\mathcal{M}}\right] \wedge x \in \dot{E}^{\mathcal{M}}, "
$$


and so by considering the $\mathcal{L}_{\mathrm{pm}}\left(\left\{\dot{x}_{i}: i \in \omega\right\}\right)$-sentence "there exists a unique $x$ with $\psi\left(x, \dot{x}_{1}, \ldots, \dot{x}_{k}\right) \wedge x \in \dot{E} "$,

$\mathcal{P} \models " \rho(x)$ is the unique element satisfying $\psi\left[\rho(x), \dot{x}_{1}^{\mathcal{P}}, \ldots, \dot{x}_{k}^{\mathcal{P}}\right] \wedge \rho(x) \in \dot{E}^{\mathcal{P}}$,

hence $\rho(x) \in \dot{E}^{\mathcal{P}}$. If $x \notin \dot{E}^{\mathcal{M}}$, we have $x \notin \dot{E}^{\mathcal{P}}$ by the same argument.

The argument for the other predicates is analogous; hence, all predicates are interpreted the same way. Therefore, $\mathcal{N}^{M}$ and $\mathcal{N}^{P}$ are indeed isomorphic.

We will now set a general context in which we prove the following two lemmas, as we want to apply them in both the proof of Theorem 3.1 and the proof of Lemma 3.8 below, for different formulae $\varphi$.

Definition 3.3. Let $X \in \mathcal{P}_{\omega_{1}}(\mathbb{R})$ and let $\mathcal{N}$ be a countable $X$-premouse. Let $\varphi$ be an $\mathcal{L}_{\mathrm{pm}}$-formula without free variables.

1. We say $\mathcal{N}$ is a $\varphi$-witness if, and only if, $\mathcal{N}$ is 1 -small, sound, and solid, $\mathcal{N} \vDash Z F$, there are no Woodin cardinals in $\mathcal{N}$, and $\mathcal{N} \vDash \varphi$.

2. We say $\mathcal{N}$ is a minimal $\varphi$-witness if, and only if, $\mathcal{N}$ is a $\varphi$-witness and no proper initial segment of $\mathcal{N}$ is a $\varphi$-witness, i.e., whenever $\mathcal{P}$ is a proper initial segment of $\mathcal{N}$ satisfying ZF + "there are no Woodin cardinals," then $\mathcal{P} \not \models \varphi$.

Lemma 3.4. Let $X \in \mathcal{P}_{\omega_{1}}(\mathbb{R})$ and suppose that $\mathcal{N}$ is a countable $\Pi_{2}^{1}$-iterable $X$ premouse which is a minimal $\varphi$-witness for some $\mathcal{L}_{\mathrm{pm}}$-formula $\varphi$. Moreover, assume that there is another countable $X$-premouse $\mathcal{M}$ which is a $\varphi$-witness and $\omega_{1}$-iterable. Then $\mathcal{N}$ is in fact $\omega_{1}$-iterable.

Proof. Let $x$ be a real coding $\mathcal{M}$ and $\mathcal{N}$ and consider the coiteration of $\mathcal{M}$ and $\mathcal{N}$ inside $M_{1}^{\sharp}(x)$ in the sense of Lemma 2.5 using $\omega_{1}$-iterability for $\mathcal{M}$ and $\Pi_{2}^{1}$ iterability for $\mathcal{N}$. Let $\mathcal{T}$ and $\mathcal{U}$ be the resulting iteration trees on $\mathcal{M}$ and $\mathcal{N}$ with final models $\mathcal{M}^{*}$ and $\mathcal{N}^{*}$, respectively. We will show that $\mathcal{N}$ cannot win this comparison, i.e., $\mathcal{N}^{*} \unlhd \mathcal{M}^{*}$ and there is no drop on the main branch through $\mathcal{U}$. This implies that $\mathcal{N}$ is elementarily embeddable into the $\omega_{1}$-iterable premouse $\mathcal{M}^{*}$ and thus $\omega_{1}$-iterable.

Assume first that $\mathcal{M}^{*}=\mathcal{N}^{*}$, there is no drop on the main branch through $\mathcal{T}$, and there is at least one drop on the main branch through $\mathcal{U}$. Then $\mathcal{M}^{*}$ is (by elementarity) a model of ZF, contrary to the fact that $\rho_{\omega}\left(\mathcal{N}^{*}\right)<\mathcal{N}^{*} \cap$ Ord.

Finally, assume, again towards a contradiction, that $\mathcal{M}^{*} \triangleleft \mathcal{N}^{*}$ and there is no drop on the main branch through $\mathcal{T}$. The model $\mathcal{N}^{*}$ need not be fully well founded, but this does not affect the rest of the argument as we shall work in the well-founded part of $\mathcal{N}^{*}$.

Notice that $\mathcal{M}^{*}$ is a proper initial segment of $\mathcal{N}^{*}$ which (by elementarity) satisfies ZF, "there are no Woodin cardinals," and $\varphi$. Therefore, it cannot be that there is no drop in model on the main branch through $\mathcal{U}$, by elementarity and the minimality of $\mathcal{N}$. Assume for simplicity that there is exactly one drop in model on the main branch through $\mathcal{U}$, say, at level $\beta+1<\lambda$ (the general case is similar: if there is more than one drop, we repeat the argument). Using the notation from [35, Section 3.1], the fact that there is a drop in model at stage $\beta+1$ implies that $\mathcal{M}_{\beta+1}^{*}$ is a proper initial segment of $\mathcal{M}_{\xi}^{\mathcal{U}}$, where $\xi$ is the $U$-predecessor of $\beta+1$ and $\mathcal{M}_{\beta+1}^{*}$ is the model to which the next extender on the main branch through $\mathcal{U}$ is applied. So by 
elementarity between $\mathcal{M}_{\beta+1}^{*}$ and $\mathcal{N}^{*}$, there is an ordinal $\alpha^{*}$ witnessing the failure of the minimality property for $\mathcal{M}_{\beta+1}^{*}$, i.e., the following hold:

1. $\alpha^{*}<\mathcal{M}_{\beta+1}^{*} \cap \operatorname{Ord}<\mathcal{M}_{\xi}^{\mathcal{U}} \cap$ Ord,

2. $\mathcal{M}_{\beta+1}^{*} \mid \alpha^{*}$ is a model of ZF with no Woodin cardinals, and

3. $\mathcal{M}_{\beta+1}^{*} \mid \alpha^{*} \vDash \varphi$.

But $\mathcal{M}_{\beta+1}^{*}$ is an initial segment of $\mathcal{M}_{\xi}^{\mathcal{U}}$, so the same holds for $\mathcal{M}_{\xi}^{\mathcal{U}} \mid \alpha^{*}$. Now by elementarity again - this time between $\mathcal{N}$ and $\mathcal{M}_{\xi}^{\mathcal{U}}$ - this failure of the minimality property also holds for $\mathcal{N}$, contradicting the fact that $\mathcal{N}$ is a minimal $\varphi$-witness.

Lemma 3.5. Let $X \in \mathcal{P}_{\omega_{1}}(\mathbb{R})$ and let $\mathcal{M}$ and $\mathcal{N}$ be $\omega_{1}$-iterable countable $X$ premice which are minimal $\varphi$-witnesses for some $\mathcal{L}_{\mathrm{pm}}$-formula $\varphi$. Then $\mathcal{M}$ and $\mathcal{N}$ have a common iterate and on both sides of the iteration there is no drop in model on the main branch through the iteration tree.

Proof. Let $\mathcal{T}$ and $\mathcal{U}$ be the iteration trees of length $\lambda+1$ for some ordinal $\lambda$ on $\mathcal{M}$ and $\mathcal{N}$, respectively, obtained from a successful comparison in the sense of Lemma 2.4. Write $\mathcal{M}^{*}=\mathcal{M}_{\lambda}^{\mathcal{T}}$ and $\mathcal{N}^{*}=\mathcal{M}_{\lambda}^{\mathcal{U}}$ for the last models of the iteration trees. We cannot have $\mathcal{M}^{*} \triangleleft \mathcal{N}^{*}$, by the argument of Lemma 3.4. Similarly, the alternative $\mathcal{N}^{*} \triangleleft \mathcal{M}^{*}$ leads to a contradiction, so we must have $\mathcal{N}^{*}=\mathcal{M}^{*}$.

Only one side of the comparison can drop; assume that there is a drop in model on the main branch through $\mathcal{U}$. The case that the main branch through $\mathcal{T}$ drops is analogous. As in the proof of Lemma 3.4, we assume for simplicity that there is exactly one drop in model along the main branch through $\mathcal{U}$, say at stage $\beta+1<\lambda$; the general case is dealt with similarly by repeating the argument. By elementarity, $\mathcal{M}^{*}=\mathcal{N}^{*}$ and $\mathcal{M}_{\beta+1}^{*}$ are $\varphi$-witnesses. Moreover, as $\mathcal{N}$ is a minimal $\varphi$-witness, by elementarity, the same holds for $\mathcal{M}_{\xi}^{\mathcal{U}}$, where $\xi$ is as in the proof of Lemma 3.4 the $U$-predecessor of $\beta+1$. But $\mathcal{M}_{\beta+1}^{*} \triangleleft \mathcal{M}_{\xi}^{\mathcal{U}}$, contradicting the minimality property for $\mathcal{M}_{\xi}^{\mathcal{U}}$. Therefore, both sides of the comparison do not drop in model.

We are now going to define a collection of games of length $\omega^{2}$ which are generalizations of the game in [16, Lemma 3]. The argument there goes back to ideas in [4] allowing one of the two players in the game to play the theory of a model with certain properties in addition to the usual moves. In the proofs of Theorem 3.1 and Lemma 3.8 below we will consider two different instances of games from this collection where Player I plays a complete and consistent theory in the language of premice with additional constant symbols.

Before we give the definition of the games, recall that if $X \in \mathcal{P}_{\omega_{1}}(\mathbb{R})$ and $\mathcal{M}$ is an $X$-premouse, then analogously to the existence of a definable well order in $L$, there is a uniformly definable $X$-parameterized family of well orders the union of whose ranges is $\mathcal{M}$ (cf. [33, Proposition 2.4]). More specifically, we can fix a formula $\theta(\cdot, \cdot, \cdot)$ in the language of premice $\mathcal{L}_{\text {pm }}$ such that for any such $X$ and any $X$-premouse $\mathcal{M}$, the following hold:

(i) for any $x \in \mathcal{M}$, there is some $\alpha \in \operatorname{Ord}^{\mathcal{M}}$ and some $r \in X$ such that

$$
\mathcal{M} \models \theta(\alpha, r, x) ;
$$


(ii) for all $r \in X$ and $\alpha \in \operatorname{Ord}^{\mathcal{M}}$ there is at most one $x \in \mathcal{M}$ such that

$$
\mathcal{M} \models \theta(\alpha, r, x) \text {. }
$$

Moreover, fix recursive bijections $m$ and $n$ assigning an odd number $>1$ to each $\mathcal{L}_{\mathrm{pm}}\left(\left\{\dot{x}_{i}: i \in \omega\right\}\right)$-formula $\varphi$ such that $m$ and $n$ have disjoint recursive ranges and for every $\varphi, m(\varphi)$ and $n(\varphi)$ are larger than $\max \left\{i: \dot{x}_{i}\right.$ occurs in $\left.\varphi\right\}$.

Definition 3.6. Let $\varphi$ and $\psi\left(x_{0}, a, b\right)$ be $\mathcal{L}_{\mathrm{pm}}$-formulae and let $\mathcal{G}_{\varphi, \psi}$ denote the following game of length $\omega^{2}$ on $\omega$ : Fix some enumeration $\left(\phi_{i}: i \in \omega\right)$ of all $\mathcal{L}_{\mathrm{pm}}\left(\left\{\dot{x}_{i}: i \in \omega\right\}\right)$-formulae such that $\dot{x}_{i}$ does not appear in $\phi_{j}$ if $j \leq i$. Then a typical run of $\mathcal{G}_{\varphi, \psi}$ looks as follows:

$$
\begin{array}{c|ccccc}
\mathrm{I} & x_{0} & a & v_{0}, x_{1} & v_{1}, x_{3} & \cdots \\
\hline \mathrm{II} & b & x_{2} & x_{4} & \cdots
\end{array}
$$

1. Player I starts by playing some parameter $x_{0} \in{ }^{\omega} \omega$;

2. Players I and II take turns playing natural numbers to construct reals $a, b \in$ ${ }^{\omega} \omega$;

3. Players I and II take turns, respectively playing sequences of natural numbers $\left(v_{i}, x_{2 i+1}\right)$ and $x_{2 i+2}$ in ${ }^{\omega} \omega$, for $i \in \omega$. We ask that $v_{i} \in\{0,1\}$.

Here $v_{i}$ will be interpreted as the truth value of the formula $\phi_{i}$ from the enumeration fixed above. This can be thought of as Player I either accepting or rejecting the formula $\phi_{i}$. If so, the play determines a complete theory $T$ in the language $\mathcal{L}_{\mathrm{pm}}\left(\left\{\dot{x}_{i}: i \in \omega\right\}\right)$.

Player I wins the game $\mathcal{G}_{\varphi, \psi}$ if, and only if,

(1) $x_{1}=a \oplus b$.

(2) For each $i \in \omega, T$ contains the sentence $\dot{x}_{i} \in{ }^{\omega} \omega$ and, moreover, for each $j, m \in \omega, T$ contains the sentence $\dot{x}_{i}(m)=j$ if, and only if, $x_{i}(m)=j$.

(3) For every formula $\phi(x)$ with one free variable in the expanded language $\mathcal{L}_{\mathrm{pm}}\left(\left\{\dot{x}_{i}: i \in \omega\right\}\right)$, and $m(\phi)$ and $n(\phi)$ as fixed above, $T$ contains the statements

$$
\begin{gathered}
\exists x \phi(x) \rightarrow \exists x \exists \alpha\left(\phi(x) \wedge \theta\left(\alpha, \dot{x}_{m(\phi)}, x\right)\right), \\
\exists x(\phi(x) \wedge x \in \dot{X}) \rightarrow \phi\left(\dot{x}_{n(\phi)}\right) .
\end{gathered}
$$

(4) $T$ is a complete, consistent theory such that for every countable model $\mathcal{M}$ of $T$ and every model $\mathcal{N}^{*}$ which is the definable closure of $\left\{x_{i}: i<\omega\right\}$ in $\mathcal{M} \uparrow \mathcal{L}_{\mathrm{pm}}, \mathcal{N}^{*}$ is well founded and if $\mathcal{N}$ denotes the transitive collapse of $\mathcal{N}^{*}$,
(a) $\mathcal{N}$ is an $X$-premouse, where $X=\left\{x_{i}: i \in \omega\right\}$,
(b) $\mathcal{N}$ is a minimal $\varphi$-witness,
(c) $\mathcal{N}$ is $\Pi_{2}^{1}$-iterable in the sense of [30, Definition 1.6], and
(d) $\mathcal{N} \vDash \psi\left(x_{0}, a, b\right)$.

If Player I plays according to all these rules, he wins the game. In this case there is a unique premouse $\mathcal{N}_{p}$ as in (4) associated to the play $p=\left(x_{0}, a \oplus b, v_{0}, x_{1}, x_{2}, \ldots\right)$ of the game. Otherwise, Player II wins.

REMARK 3.7. Rule (3) in the game $\mathcal{G}_{\varphi, \psi}$ ensures that if $\mathcal{M}$ is a model of the theory $T$, then the definable closure of $\left\{x_{i}: i \in \omega\right\}$ in $\mathcal{M} \uparrow \mathcal{L}_{\text {pm }}$ is an elementary 
substructure of $\mathcal{M} \uparrow \mathcal{L}_{\mathrm{pm}}$ (by the Tarski-Vaught criterion) by the following argument: Suppose $\exists x \phi(x)$ holds in $\mathcal{M}$. Then rule (3) ensures that $\exists x \exists \alpha(\phi(x) \wedge$ $\left.\theta\left(\alpha, \dot{x}_{m(\phi)}, x\right)\right)$. Now, the formula $\phi(x) \wedge \exists \alpha\left(\theta\left(\alpha, \dot{x}_{m(\phi)}, x\right) \wedge \forall \beta \in \alpha \neg \exists y(\phi(y) \wedge\right.$ $\left.\left.\theta\left(\beta, \dot{x}_{m(\phi)}, y\right)\right)\right)$ uniquely defines a witness $x$ for $\phi(x)$ (the minimal witness according to the well order given by $\left.\theta\left(\cdot, \dot{x}_{m(\phi)}, \cdot\right)\right)$. Hence, rule (4) can be followed by Player I by playing an appropriate theory $T$, as then the model $\mathcal{N}$ is uniquely determined by it, by Lemma 3.2 .

To prove Theorem 3.1, we first need to show the following lemma. We thank John Steel for pointing out to us that it can be proved via a modification of our argument for Theorem 3.1.

LEMmA 3.8. Suppose that $\boldsymbol{\Pi}_{2}^{1}$ games of length $\omega^{2}$ are determined. Then there is a club $\mathcal{C}^{*} \subset \mathcal{P}_{\omega_{1}}(\mathbb{R})$ such that for all $A \in \mathcal{C}^{*}$,

$$
\mathbb{R} \cap M_{1}(A)=A .
$$

Proof. Assume towards a contradiction that the statement of the lemma fails. Thus, there is a stationary set of sets $A \in \mathcal{P}_{\omega_{1}}(\mathbb{R})$ such that

$$
A \subsetneq \mathbb{R} \cap M_{1}(A) .
$$

Let

and

$$
\varphi \equiv \text { "there is a real } y \text { which is not in } \dot{X} "
$$

$$
\begin{gathered}
\psi\left(x_{0}, a, b\right) \equiv \text { "there is a real definable from } x_{0} \text { which is not in } \dot{X} \text { and } \\
\text { if } z_{0} \text { is the least real definable from } x_{0} \text { which is not in } \dot{X}, \\
\text { then its } b_{0} \text { th digit is } a_{1} \text { ", }
\end{gathered}
$$

where $a=\left(a_{0}, a_{1}, \ldots\right)$ and $b=\left(b_{0}, b_{1}, \ldots\right)$ with $a_{i}, b_{i} \in \omega$ for all $i \in \omega$. This will only be applied in $X$-premice $\mathcal{M}$ for some $X \in \mathcal{P}_{\omega_{1}}(\mathbb{R})$ with $x_{0} \in X$ and "least" refers to the least real in the well order of elements of $\mathcal{M}$ definable from $x_{0}$ which is given by $\theta\left(\cdot, x_{0}, \cdot\right)$.

Consider the game $\mathcal{G}_{\varphi, \psi}$, i.e., after Player I plays the parameter $x_{0}$, the only relevant moves are the following: Player II plays a natural number $b_{0}$ asking Player I for the $b_{0}$ th digit of the least real definable from $x_{0}$ which is not going to be in $X$ and Player II answers by playing $a_{1}$. Afterwards they continue playing the rest of $X=\left\{x_{0}, x_{1}, \ldots\right\}$ and the theory of a $\varphi$-witness.

The winning condition in this game $\mathcal{G}_{\varphi, \psi}$ is $\Pi_{2}^{1}$, so it is determined and we can distinguish the following two cases to obtain a contradiction by arguing that no player can have a winning strategy.

CASE 1. Player I has a winning strategy $\sigma$ in $\mathcal{G}_{\varphi, \psi}$.

Let $W$ be the transitive collapse of a countable elementary substructure $Y$ of some large $V_{\kappa}$ such that $\sigma \in Y$ and let $\pi$ denote the inverse of the collapse embedding, i.e.,

$$
\pi: W \cong Y \prec V_{\kappa} .
$$

Since $\mathbb{R}^{W}$ is countable, it follows that $M_{1}^{\sharp}\left(\mathbb{R}^{W}\right)$ exists and is $\omega_{1}$-iterable. Since the set of $\mathbb{R}^{W}$ for such elementary substructures $W$ is a club in $\mathcal{P}_{\omega_{1}}(\mathbb{R})$, we may assume that

$$
\mathbb{R}^{W} \subsetneq \mathbb{R} \cap M_{1}\left(\mathbb{R}^{W}\right)
$$


The game $\mathcal{G}_{\varphi, \psi}$ can be defined in $W$. Let $\bar{\sigma} \in W$ be such that $\pi(\bar{\sigma})=\sigma$, i.e., $\bar{\sigma}=\sigma \cap W$. By elementarity,

$$
W \models " \bar{\sigma} \text { is a winning strategy for Player I in } \mathcal{G}_{\varphi, \psi} . "
$$

Let $h$ be a well ordering of $\mathbb{R}^{W}$ in $V$ of order-type $\omega$. Consider a play of the game $\mathcal{G}_{\varphi, \psi}$ in $V$ in which Player II plays some $b \in{ }^{\omega} \omega$ and $x_{2}, x_{4}, \ldots$ according to $h$ and Player I plays according to the winning strategy $\sigma$. Every proper initial segment of the play is in the domain of $\bar{\sigma}$. It follows that the real part $\left(x_{0}, x_{1}, x_{2}, \ldots\right)$ of the play, say $p$, enumerates $\mathbb{R}^{W}$. Furthermore, $p$ is consistent with $\sigma$, whereby $p$ is won by Player I. This means that $p$ determines a $\Pi_{2}^{1}$-iterable $\mathbb{R}^{W}$-premouse $\mathcal{N}_{p}$ which is a minimal $\varphi$-witness.

Let $\delta_{\mathbb{R}^{W}}$ denote the Woodin cardinal in $M_{1}\left(\mathbb{R}^{W}\right)$. Since we chose $W$ so that $\mathbb{R}^{W} \subsetneq$ $\mathbb{R} \cap M_{1}\left(\mathbb{R}^{W}\right) \mid \delta_{\mathbb{R}^{W}}$ and since satisfying $\varphi$ for the $\mathbb{R}^{W}$-premice $\mathcal{N}_{p}$ and $M_{1}\left(\mathbb{R}^{W}\right) \mid \delta_{\mathbb{R}^{W}}$ means having a real which is not in $\mathbb{R}^{W}$, we have that $M_{1}\left(\mathbb{R}^{W}\right) \mid \delta_{\mathbb{R}^{W}}$ is an $\omega_{1}$-iterable $\varphi$-witness. Thus, Lemma 3.4 implies that $\mathcal{N}_{p}$ is $\omega_{1}$-iterable as well.

Let $x_{0} \in{ }^{\omega} \omega$ be the first move given by $\sigma$ (so $x_{0}$ is also the first move given by $\bar{\sigma}$ ). Moreover, let $a_{0} \in \omega$ be the first move of Player I after $x_{0}$ given by $\sigma($ and $\bar{\sigma})$. Let $\tau$ be the real defined by

$$
\tau(n)=\bar{\sigma}\left(x_{0}, a_{0}, n\right),
$$

for all possible moves $n \in \omega$ of Player II for $b_{0}$. We claim that $\tau$ is the least real in $\mathcal{N}_{p}$ not in $\mathbb{R}^{W}$ which is definable from $x_{0}$. This will be a contradiction, since $\tau \in W$ as $\bar{\sigma} \in W$ and $x_{0} \in W$.

Let $\tau^{\prime}$ be the least real in $\mathcal{N}_{p}$ not in $\mathbb{R}^{W}$ which is definable from $x_{0}$. Assume that $\tau^{\prime} \neq \tau$ and choose some $n_{0} \in \omega$ such that $\tau\left(n_{0}\right) \neq \tau^{\prime}\left(n_{0}\right)$. Let $q$ be the play of the game $\mathcal{G}_{\varphi, \psi}$ in which Player I plays according to $\bar{\sigma}$ and Player II plays some $b \in{ }^{\omega} \omega$ with first digit $n_{0}$ and then $h$ as above. As Player I plays according to $\bar{\sigma}$ and hence according to $\sigma$, this is a winning play for Player I. Let $\mathcal{N}_{q}$ be the corresponding model. In particular, $\mathcal{N}_{q} \vDash \psi\left(x_{0}, a, b\right)$, i.e., the least real in $\mathcal{N}_{q}$ not in $\mathbb{R}^{W}$ which is definable from $x_{0}$ has $a_{1}=\bar{\sigma}\left(x_{0}, a_{0}, n_{0}\right)$ as $n_{0}$ th digit.

By the rules of the game, $\mathcal{N}_{q}$ is a $\Pi_{2}^{1}$-iterable $\mathbb{R}^{W}$-premouse which is a minimal $\varphi$-witness. Hence, Lemma 3.4 yields (as in the case of $\mathcal{N}_{p}$ ) that $\mathcal{N}_{q}$ is in fact $\omega_{1}$ iterable. So Lemma 3.5 implies that $\mathcal{N}_{p}$ and $\mathcal{N}_{q}$ coiterate to a common model and there is no drop in model on the main branch through the trees on both sides of the coiteration. By definition, both $\mathcal{N}_{p}$ and $\mathcal{N}_{q}$ are pointwise definable from $\mathbb{R}^{W}$. Therefore it is easy to see that in fact $\mathcal{N}_{p}=\mathcal{N}_{q}$. In particular, $\mathcal{N}_{p}$ and $\mathcal{N}_{q}$ have the same least real $\tau^{\prime}$ definable from $x_{0}$ which is different from all reals in $\mathbb{R}^{W}$ and by choice of $q, \tau^{\prime}\left(n_{0}\right)=\bar{\sigma}\left(x_{0}, a_{0}, n_{0}\right)=\tau\left(n_{0}\right)$, which is the desired contradiction.

CASE 2. Player II has a winning strategy $\sigma$ in $\mathcal{G}_{\varphi, \psi}$.

Let $W$ be the transitive collapse of a countable elementary substructure $Y$ of some large $V_{\kappa}$ such that $\sigma \in Y$ and let $\pi$ denote the inverse of the collapse embedding. Moreover, let $\bar{\sigma} \in W$ be such that $\pi(\bar{\sigma})=\sigma$, i.e., $\bar{\sigma}=\sigma \cap W$. Since $\mathbb{R}^{W}$ is countable, it follows that $M_{1}^{\sharp}\left(\mathbb{R}^{W}\right)$ exists and is $\omega_{1}$-iterable in $V$. As before, by our hypothesis we may assume that

$$
\mathbb{R}^{W} \subsetneq \mathbb{R} \cap M_{1}\left(\mathbb{R}^{W}\right)
$$


Let $\mathcal{Q}=M_{1}\left(\mathbb{R}^{W}\right) \mid \alpha$, where $\alpha$ is least such that $\mathcal{Q} \vDash \mathrm{ZF}+$ "there are no Woodin cardinals" and $\mathcal{Q}$ contains a real which is not in $\mathbb{R}^{W}$. Let $\mathcal{N}^{*, \mathcal{Q}}$ be the definable closure of $\mathbb{R}^{W}$ in $\mathcal{Q}$ and $\mathcal{N}^{\mathcal{Q}}$ the transitive collapse of $\mathcal{N}^{*, \mathcal{Q}}$. Then $\mathcal{N}^{\mathcal{Q}} \prec \mathcal{Q}$. Thus, there is some real $z$ in $\mathcal{N}^{\mathcal{Q}}$ which is not in $\mathbb{R}^{W}$ such that $z$ is definable in $\mathcal{N}^{\mathcal{Q}}$ from some real $x_{0} \in \mathbb{R}^{W}$. We shall ask Player I to begin every play of the game $\mathcal{G}_{\varphi, \psi}$ by playing this real $x_{0}$. Assume without loss of generality that $z$ is the least real in $\mathcal{N}^{\mathcal{Q}} \backslash \mathbb{R}^{W}$ definable from $x_{0}$ according to the well order defined by $\theta\left(\cdot, x_{0}, \cdot\right)$.

Consider the play $p$ in $\mathcal{G}_{\varphi, \psi}$ in which Player II plays according to $\bar{\sigma}$ (and hence according to the winning strategy $\sigma$ ) and Player I plays:

(1) $x_{0} \in \mathbb{R}^{W}$, in the first round,

(2) $a_{1}=z\left(b_{0}\right)$, in response to Player II playing $b_{0} \in \omega$ according to $\sigma$,

(3) other, arbitrary, natural numbers $a_{0}, a_{2}, a_{3}, \ldots$,

(4) some enumeration $h$ of $\mathbb{R}^{W}$ in order-type $\omega$ with $h \in V$ as in Case 1, together with the theory of $\mathcal{Q}$ in the language $\mathcal{L}_{\mathrm{pm}}\left(\left\{\dot{x}_{i}: i \in \omega\right\}\right)$, where the constants $\dot{x}_{i}$ are interpreted by the reals $x_{i} \in \mathbb{R}^{W}$ according to $p$, satisfying rules (1), (2), and (3) of the game $\mathcal{G}_{\varphi, \psi}$.

Arguing as before, one shows that the reals played in $p$ enumerate $\mathbb{R}^{W}$. It follows that the model $\mathcal{N}^{\mathcal{Q}}$ witnesses that $p$ is a winning play for Player I, which contradicts the fact that $\sigma$ is a winning strategy for Player II. This proves the lemma.

REMARK 3.9. It is also possible to prove Lemma 3.8 with the following variant of the argument we gave above. Instead of playing a code for a theory via $v_{i} \in\{0,1\}$ for $i \in \omega$, we could ask Player I to play a (fine structural) code for a premouse $\mathcal{N}$ projecting to $\omega$ digit by digit via $v_{i} \in \omega$ for $i \in \omega$. In addition, we can let Player I play $w_{i} \in \omega$ together with $v_{i}$ for each $i \in \omega$ to play another real $w$ digit by digit. Then we say Player I wins if, and only if, the premouse $\mathcal{N}$ he codes satisfies (a)-(c) in Definition 3.6 where we no longer require ZF in the definition of $\varphi$-witness, but ask that $w$ is the minimal (in the natural order on formulae and ordinal parameters) real definable from $x_{0}$ over $\mathcal{N}$ which is not in $X=\left\{x_{i}: i \in \omega\right\}$ and that no proper initial segment of $\mathcal{N}$ satisfies this property. Then a similar argument as in Lemma 3.4 shows that if there is an $\omega_{1}$-iterable model with this property, $\mathcal{N}$ is in fact $\omega_{1}$-iterable as well. Now a similar argument as in the proof of Lemma 3.8 above shows that this game works. Moreover, the same idea can be used to phrase the proof of Theorem 3.1 below differently.

Finally, we are ready to prove Theorem 3.1.

Proof of Theorem 3.1. Assume towards a contradiction that we have

$$
M_{1}(B) \mid \delta_{B} \forall \mathrm{AD}
$$

for a stationary set of sets $B \in \mathcal{P}_{\omega_{1}}(\mathbb{R})$, where $\delta_{B}$ denotes the Woodin cardinal in $M_{1}(B)$. Let

$$
\varphi \equiv “ \dot{X}=\mathbb{R}+\neg \mathrm{AD} "
$$

and

$$
\begin{gathered}
\psi\left(x_{0}, a, b\right) \equiv \text { "there is a nondetermined set of reals definable from } x_{0} \text { and } \\
\text { if } Z \text { is the least such set in the well-order relative to } \dot{X}, \\
\text { then } a \oplus b \in Z \text { ". }
\end{gathered}
$$


This will only be applied in $X$-premice $\mathcal{M}$ for some $X \in \mathcal{P}_{\omega_{1}}(\mathbb{R})$ with $x_{0} \in X$ and as in the proof of Lemma 3.8 "least" refers to the least set in the well order of elements of $\mathcal{M}$ definable from $x_{0}$ which is given by $\theta\left(\cdot, x_{0}, \cdot\right)$.

In this case, the game $\mathcal{G}_{\varphi, \psi}$ is a variant of the Kechris-Solovay game in [11] (see also the game in [21, Lemma 2.3]) adapted as a model game. The winning condition is $\Pi_{2}^{1}$, so the game $\mathcal{G}_{\varphi, \psi}$ is determined. We will obtain a contradiction by arguing that no player can have a winning strategy.

CASE 1. Player I has a winning strategy $\sigma$ in $\mathcal{G}_{\varphi, \psi}$.

Let $W$ be the transitive collapse of a countable elementary substructure $Y$ of some large $V_{\kappa}$ with $\sigma \in Y$ and let $\pi$ denote the inverse of the collapse embedding, i.e.,

$$
\pi: W \cong Y \prec V_{\kappa} .
$$

Since $\mathbb{R}^{W}=\mathbb{R} \cap W$ is countable, it follows that $M_{1}^{\sharp}\left(\mathbb{R}^{W}\right)$ exists and is $\omega_{1}$-iterable (in $V$ ). By Lemma 3.8, the set of $\mathbb{R}^{W}$ for $W$ as above with the additional property that $M_{1}\left(\mathbb{R}^{W}\right) \cap \mathbb{R}=\mathbb{R}^{W}$ is club in $\mathcal{P}_{\omega_{1}}(\mathbb{R})$. By assumption, we may thus choose $W$ so that $M_{1}\left(\mathbb{R}^{W}\right) \cap \mathbb{R}=\mathbb{R}^{W}$ and in addition

$$
M_{1}\left(\mathbb{R}^{W}\right) \mid \delta_{\mathbb{R}^{W}} \forall \neq \mathrm{AD} .
$$

Note that the game $\mathcal{G}_{\varphi, \psi}$ can be defined in $W$ and let $\bar{\sigma} \in W$ be such that $\pi(\bar{\sigma})=\sigma$, i.e., $\bar{\sigma}=\sigma \cap W$. By elementarity,

$$
W \models " \bar{\sigma} \text { is a winning strategy for Player I in } \mathcal{G}_{\varphi, \psi} . "
$$

Let $h$ be a well-ordering of $\mathbb{R}^{W}$ in $V$ of order-type $\omega$. Clearly, every proper initial segment of $h$ is in $W$. Consider a play of the game $\mathcal{G}_{\varphi, \psi}$ in $V$ in which Player II plays some arbitrary $b^{*} \in{ }^{\omega} \omega$ and $x_{2}, x_{4}, \ldots$ according to $h$ and Player I plays according to the winning strategy $\sigma$. Every proper initial segment of the play is in the domain of $\bar{\sigma}$. It follows that the real part $\left(x_{0}, x_{1}, x_{2}, \ldots\right)$ of the play, say $p$, enumerates $\mathbb{R}^{W}$. Futhermore, $p$ is consistent with $\sigma$, whereby $p$ is won by Player I. This means that $p$ determines a $\Pi_{2}^{1}$-iterable $\mathbb{R}^{W}$-premouse $\mathcal{N}_{p}$ which is a minimal $\varphi$-witness. In particular, $\mathcal{N}_{p} \cap \mathbb{R}=\mathbb{R}^{W}$. Since both $\mathcal{N}_{p}$ and $M_{1}\left(\mathbb{R}^{W}\right) \mid \delta_{\mathbb{R}^{W}}$ are $\mathbb{R}^{W}$-premice and $M_{1}\left(\mathbb{R}^{W}\right) \mid \delta_{\mathbb{R}^{W}}$ is a $\varphi$-witness, $\mathcal{N}_{p}$ is $\omega_{1}$-iterable by Lemma 3.4 .

Let $x_{0} \in{ }^{\omega} \omega$ be the first move given by $\sigma$ (so $x_{0}$ is also the first move given by $\bar{\sigma})$. Let $Z=Z\left(x_{0}, \mathcal{N}_{p}\right)$ denote the least nondetermined set of reals in $\mathcal{N}_{p}$ which is definable from $x_{0}$. This exists since $\mathcal{N}_{p} \vDash \psi\left(x_{0}, a^{*}, b^{*}\right)$, where $a^{*}$ is the sequence of natural numbers Player I plays after $x_{0}$ in response to $b^{*}$ according to $\sigma$. There is a natural strategy $\tau$ for Player I in $G(Z)$ - the Gale-Stewart game with winning condition $Z$ played in $\mathcal{N}_{p}$-which is induced by $\bar{\sigma}$. Let $\tau$ be the unique strategy such that for $a, b \in{ }^{\omega} \omega \cap \mathcal{N}_{p}$,

$$
a=\tau(b) \text { if, and only if, }\left(x_{0}, a\right)=\bar{\sigma}(b) .
$$

Note that $\tau \in W$ as $\bar{\sigma}, x_{0} \in W$ and, since the reals of $\mathcal{N}_{p}$ are those of $W$, we also have $\tau \in \mathcal{N}_{p}$.

We claim that $\tau$ is a winning strategy for Player I (in the game $G(Z)$ in $\mathcal{N}_{p}$ ), which will contradict the fact that the set $Z$ is nondetermined in $\mathcal{N}_{p}$. Let $a \oplus b \in \mathbb{R}^{W}$ be a play by $\tau$. Let $q$ be the play of the game $\mathcal{G}_{\varphi, \psi}$ in which Player I plays according to $\bar{\sigma}$ and Player II plays $b$ and then $h$ as above. As Player I plays according to 
$\bar{\sigma}$ and hence according to $\sigma$, this is a winning play for Player I. Let $\mathcal{N}_{q}$ be the corresponding model. In particular, $\mathcal{N}_{q} \vDash \psi\left(x_{0}, a, b\right)$, i.e., $a \oplus b \in Z\left(x_{0}, \mathcal{N}_{q}\right)$, where $Z\left(x_{0}, \mathcal{N}_{q}\right)$ denotes the least nondetermined set of reals in $\mathcal{N}_{q}$ which is definable from $x_{0}$.

By the rules of the game, $\mathcal{N}_{q}$ is a $\Pi_{2}^{1}$-iterable $\mathbb{R}^{W}$-premouse which is a minimal $\varphi$-witness. Hence Lemma 3.4 yields that $\mathcal{N}_{q}$ is in fact $\omega_{1}$-iterable. So we can apply Lemma 3.5 to $\mathcal{N}_{q}$ and $\mathcal{N}_{p}$. In fact, $\mathcal{N}_{p}=\mathcal{N}_{q}$ as both are pointwise definable from $\mathbb{R}^{W}$. Therefore, $Z=Z\left(x_{0}, \mathcal{N}_{p}\right)=Z\left(x_{0}, \mathcal{N}_{q}\right)$ and $a \oplus b \in Z$. Hence, $\tau$ is a winning strategy for Player I, contrary to the fact that $Z$ is nondetermined in $\mathcal{N}_{p}$.

CASE 2. Player II has a winning strategy $\sigma$ in $\mathcal{G}_{\varphi, \psi}$.

As before, let $W$ be the transitive collapse of a countable elementary substructure $Y$ of some large $V_{\kappa}$ with $\sigma \in Y$ and let $\pi$ denote the inverse of the collapse embedding, i.e.,

$$
\pi: W \cong Y \prec V_{\kappa} .
$$

Then $M_{1}^{\sharp}\left(\mathbb{R}^{W}\right)$ exists and is $\omega_{1}$-iterable in $V$. As before, we may choose $W$ so that

$$
M_{1}\left(\mathbb{R}^{W}\right) \cap \mathbb{R}=\mathbb{R}^{W} \text { and } M_{1}\left(\mathbb{R}^{W}\right) \mid \delta_{\mathbb{R}^{W}} \not A \mathrm{AD} .
$$

Let $\bar{\sigma}=\sigma \cap W$, so that $\bar{\sigma} \in W$ and

$$
W \models \text { " } \bar{\sigma} \text { is a winning strategy for Player II in } \mathcal{G}_{\varphi, \psi} . "
$$

Let $\mathcal{Q}=M_{1}\left(\mathbb{R}^{W}\right) \mid \alpha$, where $\alpha$ is least such that there are no Woodin cardinals in $\mathcal{Q}$ and $\mathcal{Q}=\mathrm{ZF}+\neg \mathrm{AD}$. Let $\mathcal{N}^{*, \mathcal{Q}}$ be the definable closure of $\mathbb{R}^{W}$ in $\mathcal{Q}$ and $\mathcal{N}^{\mathcal{Q}}$ the transitive collapse of $\mathcal{N}^{*, \mathcal{Q}}$. Then $\mathcal{N}^{\mathcal{Q}} \prec \mathcal{Q}$ and $\mathcal{N}^{\mathcal{Q}}$ is $\omega_{1}$-iterable because it is elementary embedded in the $\omega_{1}$-iterable premouse $\mathcal{Q}$. Moreover, there is some nondetermined set in $\mathcal{N}^{\mathcal{Q}}$ definable from some real $x_{0} \in \mathbb{R}^{W}$. We shall ask Player I to play this real $x_{0}$ followed by some real $a$ and some enumeration $h \in V$ of $\mathbb{R}^{W}$ in order-type $\omega$ together with the theory of $\mathcal{Q}$ (of course, organized in such a way that he satisfies rules (1), (2), and (3) of the game). Let $Z\left(x_{0}, \mathcal{N}^{\mathcal{Q}}\right)$ be as before.

Since $\bar{\sigma}$ is a winning strategy for Player II in the game $\mathcal{G}_{\varphi, \psi}$ in $W$, in particular $\bar{\sigma}$ wins against all plays in which Player I begins by playing $x_{0}$. As before, there is a natural strategy $\tau \in \mathcal{N}^{\mathcal{Q}}$ for Player II for the Gale-Stewart game inside $\mathcal{N}^{\mathcal{Q}}$ with payoff set $Z\left(x_{0}, \mathcal{N}^{\mathcal{Q}}\right)$, namely, the unique strategy such that

$$
b=\tau(a) \text { if, and only if, } b=\bar{\sigma}\left(x_{0}, a\right) .
$$

A similar argument as in Case 1, using that Player I cannot lose a play $p$ as above because of having played the wrong theory, but only because $a \oplus b \notin Z\left(x_{0}, \mathcal{N}_{p}\right)$, gives that $\tau$ is a winning strategy for Player II in $\mathcal{N}^{\mathcal{Q}}$. This finishes the proof of Theorem 3.1.

$\S 4$. Dependent choice, scales, and mouse capturing. By Theorem 3.1 we obtain a countable set of reals $A$ such that $M_{1}(A)$ is an $A$-premouse constructed over its reals and $M_{1}(A) \vDash \mathrm{ZF}+\mathrm{AD}$ from the assumption that all $\Pi_{2}^{1}$ games of length $\omega^{2}$ are determined. We aim to show that there is a model with $\omega+1$ Woodin cardinals from this hypothesis. Before we do that in the next section, we first show some structural properties of this model. 
First, we have that in fact $M_{1}(A) \vDash D C$ by the following theorem, which is a special case of [19, Theorem 1.1] (building on [33] and [9]).

THEOREM 4.1. Let $A \in \mathcal{P}_{\omega_{1}}(\mathbb{R})$ be a countable set of reals such that $M_{1}(A) \cap \mathbb{R}=A$ and $M_{1}(A) \vDash \mathrm{ZF}+\mathrm{AD}$. Then $M_{1}(A) \vDash \mathrm{DC}$.

In what follows we argue that, in $M_{1}(A), \Sigma_{1}^{2}$ has the scale property and $\Theta=\theta_{0}$, i.e., the Solovay sequence is trivial. Assuming AD, recall that

$$
\Theta=\sup \{\beta \text { : there is a surjection } f: \mathbb{R} \rightarrow \beta\},
$$

and

$$
\theta_{0}=\sup \{\beta: \text { there is an OD surjection } f: \mathbb{R} \rightarrow \beta\} .
$$

These properties of $M_{1}(A)$ have proofs similar to those for $L(\mathbb{R})$. For example, using [33] the scale analysis of $L(\mathbb{R})$ from [34] can be done inside $M_{1}(A)$ and yields that $\Sigma_{1}^{M_{1}(A)}$ has the scale property. Moreover, as in $L(\mathbb{R})$, it is easy to see that $\left(\Sigma_{1}^{2}\right)^{M_{1}(A)}=\Sigma_{1}^{M_{1}(A)}$. Similarly, $\left(\Theta=\theta_{0}\right)^{M_{1}(A)}$. In fact, by generalizing the arguments used for $L(\mathbb{R})$, we can also get that $\mathrm{AD}^{+}$holds in $M_{1}(A)$ but we will not need that. We summarize this in the next theorem.

THEOREM 4.2. Let $A \in \mathcal{P}_{\omega_{1}}(\mathbb{R})$ be a countable set of reals such that $M_{1}(A) \cap \mathbb{R}=A$ and $M_{1}(A) \vDash \mathrm{ZF}+\mathrm{AD}$. Then $M_{1}(A) \vDash " \Sigma_{1}^{2}$ has the scale property" $+\Theta=\theta_{0}$.

Finally, we also have that $M_{1}(A)$ satisfies Mouse Capturing (MC), i.e., that for any two countable transitive sets $x$ and $y$ such that $x \subseteq y$ and $x \in \mathrm{OD}_{y \cup\{y\}}, x$ is contained in an $\omega_{1}$-iterable $y$-premouse. This follows from [36, Theorem 1.5] (due to Woodin). To see this, note that in $M_{1}(A)$ as below, all mice are tame. If not, in particular $M_{\omega+2}^{\sharp}$ exists in $M_{1}(A)$ so, using [23], $\Pi_{3}^{1}$-determinacy holds for games on $\mathbb{N}$ of length $\omega^{2}$. Therefore, by [2, Lemma 4.2], $M_{1}^{\sharp}(A)$ exists inside of $M_{1}(A)$, which is a contradiction. ${ }^{5}$ So we have the following theorem.

THEOREM 4.3. Let $A \in \mathcal{P}_{\omega_{1}}(\mathbb{R})$ be a countable set of reals such that $M_{1}(A) \cap \mathbb{R}=A$ and $M_{1}(A) \vDash \mathrm{ZF}+\mathrm{AD}$. Then $M_{1}(A) \vDash \mathrm{MC}$.

§5. $\omega+1$ Woodin cardinals. In this section we will use the results from the previous sections to construct a premouse with $\omega+1$ Woodin cardinals. More precisely, we prove the following theorem:

TheOrem 5.1. Suppose there is some $A \in \mathcal{P}_{\omega_{1}}(\mathbb{R})$ such that

1. $M_{1}^{\sharp}(A)$ exists,

2. $M_{1}(A) \cap \mathbb{R}=A$, and

3. $M_{1}(A) \vDash \mathrm{AD}$.

Then there is an active premouse with $\omega+1$ Woodin cardinals.

Using Section 4, for the rest of this section, we fix a countable set of reals $A$ such that $M_{1}(A)$ is an $\omega_{1}$-iterable $A$-premouse with $M_{1}(A) \cap \mathbb{R}=A$ and

$$
M_{1}(A) \vDash \mathrm{ZF}+\mathrm{DC}+\mathrm{AD}+{ }^{~ "} \Sigma_{1}^{2} \text { has the scale property" }+\Theta=\theta_{0}+\mathrm{MC} \text {. }
$$

The rest of this section is devoted to the proof of Theorem 5.1. Most of the proof in this section closely follows ideas from Section 3 in the unpublished notes [31].

\footnotetext{
${ }^{5}$ Instead of appealing to [2] we could have also just added that all mice in $M_{1}(A)$ are tame as an extra hypothesis in Theorem 4.3 because a nontame mouse is beyond what we want to get in Theorem 1.1.
} 
See also Sections 6.5 and 6.6 in [37] for a similar argument applied to $L(\mathbb{R})$ or [26]. We start by introducing some notation, generalizing ideas from [37, Section 3] and [29] to our context. Suppose that $A$ is as in the statement of Theorem 5.1 and work inside $V=M_{1}(A)$.

The proof of Theorem 5.1 can be split in several parts. After we recall a useful standard fact, we define suitable premice. From these we will, by pseudocomparison and pseudogenericity iteration, obtain models which we can use in a Prikry-like forcing to construct a model with $\omega$ Woodin cardinals. Then we argue that this model can be rearranged into a premouse on top of which we can perform a $\mathcal{P}$-construction to add one more Woodin cardinal.

The following standard lemma will be useful later on:

LEMmA 5.2. There is a $\Sigma_{1}^{2}$ scale $\vec{\phi}$ on a $\Sigma_{1}^{2}$ set which is universal for $\Sigma_{1}^{2}$ such that, letting $T$ be the tree obtained from $\vec{\phi}$, we have for any countable transitive set $a$,

$$
\begin{aligned}
\mathcal{P}(a) \cap L[T, a] & =\mathcal{P}(a) \cap \mathrm{OD}_{a \cup\{a\}} \\
& =\left\{b \in H\left(\omega_{1}\right): b \text { belongs to an } \omega_{1} \text {-iterable a-premouse }\right\} .
\end{aligned}
$$

Proof. The second equality easily follows from MC. For the first equality, let $U \subset \mathbb{R}^{2}$ be any $\Sigma_{1}^{2}$ set that is universal for $\Sigma_{1}^{2}$. $\Sigma_{1}^{2}$ has the scale property, so let $T$ be a tree on $\omega \times \omega \times \delta_{1}^{2}$ obtained from a $\Sigma_{1}^{2}$-scale on $U$ (thus $T$ projects to $U$ ).

Now, suppose $b \in \mathcal{P}(a) \cap \mathrm{OD}_{a \cup\{a\}}$. Let $z$ be a real coding $a$. Then the set $B$ of all $\mathrm{OD}_{a \cup\{a\}}$ subsets of $a$ (coded as a real relative to $z$ ) is $\Sigma_{1}^{2}(z)$. By the MansfieldSolovay Theorem (see, for example [10, Theorem 11.1]), either $B \subseteq L[T, z]$, or $B$ contains a perfect subset. But since $B$ is countable, it is thin. Therefore $b_{z}$, the real coding $b$ relative to $z$, is in $L[T, z]$ and hence $b \in L[T, z]$. Since this holds for all $z$ which are $\operatorname{Col}(\omega, a)$-generic over $L[T, a]$, it follows that $b \in L[T, a]$.

Conversely, every real in $L[T, a]$ is definable from $a$ and ordinal parameters. This is because the reals of $L[T, a]$ do not depend on the choice of the universal set $U$ nor on the scale on $U$ (this follows, e.g., from [18, Exercise 8G.29], see also [7]). $\dashv$

Fix a $\Sigma_{1}^{2}$ set $U$ which is universal for $\Sigma_{1}^{2}$ in $V=M_{1}(A)$ and a tree $T$ as above for the rest of this section.

5.1. Suitable premice. We begin by isolating a class of models suitable for our purposes.

Definition 5.3. Suppose $b$ is a countable transitive set. We write

$$
L p(b)=\bigcup\left\{M: M \text { is a sound } \omega_{1} \text {-iterable } b \text {-premouse such that } \rho_{\omega}(M)=b\right\} .
$$

Moreover, we inductively define $L p^{1}(b)=L p(b)$,

$$
L p^{n+1}(b)=L p\left(L p^{n}(b)\right),
$$

and

$$
L p^{\omega}(b)=\bigcup_{n<\omega} L p^{n}(b)
$$

REMARK 5.4. Recall that we are working inside $M_{1}(A)$, which is a model of AD, so the club filter is an ultrafilter on $\omega_{1}$. This can be used to show that $\omega_{1}$-iterability already implies $\omega_{1}+1$-iterability (see e.g., [35, Lemma 7.11] for details), so that any 
two $\omega_{1}$-iterable $b$-premice $M$ and $N$ as in the definition of $L p(b)$ can be successfully compared and line up, i.e., $M \unlhd N$ or $N \unlhd M$. Therefore, $L p(b)$ is a well-defined premouse.

In the definitions below, let $a$ be an arbitrary countable transitive set.

Definition 5.5. We say that an $a$-premouse $M$ is suitable if, and only if, there is an ordinal $\delta$ such that

1. $M$ is a model of ZFC - "Replacement" and $M \cap \operatorname{Ord}=\sup _{n<\omega}\left(\delta^{+n}\right)^{M}$,

2. $\delta$ is the unique Woodin cardinal in $M$, and

3. $M$ is full, i.e., for every cutpoint ${ }^{6} \eta$ in $M, L p(M \mid \eta) \unlhd M$.

If $M$ is suitable, we denote its Woodin cardinal by $\delta_{M}$.

LEMmA 5.6. Let $M$ be a countable a-premouse and $x_{M}$ a real coding $M$. Then for any real $z \geq_{T} x_{M}$, the statement " $M$ is suitable" is absolute between $V$ and $L[T, z]$.

Proof. Suppose not, say $M$ is suitable in $L[T, z]$ but there is some $\eta<M \cap$ Ord and a sound $\omega_{1}$-iterable $M \mid \eta$-premouse $N$ in $V$ with $\rho_{\omega}(N)=M \mid \eta$ such that $N \nsubseteq M$. This statement is $\Sigma_{1}^{2}$ and hence such a counterexample would also exist in $L[T, z]$. By the same argument, if we suppose that $M$ is suitable in $V$, every such $\omega_{1}$-iterable $M \mid \eta$-premouse in $L[T, z]$ is also $\omega_{1}$-iterable in $V$. Hence the statement " $M$ is suitable" is absolute between $V$ and $L[T, z]$.

Definition 5.7. Let $\mathcal{T}$ be a normal iteration tree on a suitable $a$-premouse $M$ of length $<\omega_{1}^{V}$. Then we say that $\mathcal{T}$ is correctly guided if, and only if, for every limit ordinal $\lambda<\operatorname{lh}(\mathcal{T})$, if $b$ is the branch choosen through $\mathcal{T} \uparrow \lambda$ in $\mathcal{T}$, then $\mathcal{Q}(b, \mathcal{T} \uparrow \lambda)$ exists and $\mathcal{Q}(b, \mathcal{T} \uparrow \lambda) \unlhd L p(\mathcal{M}(\mathcal{T}\lceil\lambda))$.

Definition 5.8. Let $\mathcal{T}$ be a normal iteration tree on a suitable $a$-premouse $M$ of length $<\omega_{1}^{V}$. Then we say that $\mathcal{T}$ is short if, and only if, $\mathcal{T}$ is correctly guided and if $\mathcal{T}$ has limit length, then $\mathcal{Q}(\mathcal{T})$ exists, and $\mathcal{Q}(\mathcal{T}) \unlhd L p(\mathcal{M}(\mathcal{T}))$. If $\mathcal{T}$ is correctly guided but not short, then it is said to be maximal.

As in [29], we define the notion of being suitability-strict in order to make the proofs of Lemmas 5.12 and 5.13 below work.

Definition 5.9. Let $M$ be a suitable $a$-premouse and let $\mathcal{T}$ be a normal iteration tree on $M$ of length $<\omega_{1}^{V}$. Then we say that $\mathcal{T}$ is suitability-strict if, and only if, for all $\alpha<\operatorname{lh}(\mathcal{T})$,

(i) if $[0, \alpha]_{T}$ does not drop then $\mathcal{M}_{\alpha}^{\mathcal{T}}$ is suitable, and

(ii) if $[0, \alpha]_{T}$ drops then no $\mathcal{R} \unlhd \mathcal{M}_{\alpha}^{\mathcal{T}}$ is suitable.

Definition 5.10. Let $M$ be a suitable $a$-premouse. Then we say that $M$ is short tree iterable if, and only if, whenever $\mathcal{T}$ is a short tree on $M$ of length $<\omega_{1}^{V}$,

(i) $\mathcal{T}$ is suitability-strict,

(ii) if $\mathcal{T}$ has a last model, then every putative iteration tree $\mathcal{U}$ extending $\mathcal{T}$ such that $\operatorname{lh}(\mathcal{U})=\operatorname{lh}(\mathcal{T})+1$ has a well-founded last model, and

\footnotetext{
${ }^{6}$ In the case where $\eta$ is not a cutpoint, we refer to the *-transformation in [32, Section 7].
} 
(iii) if $\mathcal{T}$ has limit length, then there exists a cofinal well-founded branch $b$ through $\mathcal{T}$ such that $\mathcal{Q}(b, \mathcal{T})=\mathcal{Q}(\mathcal{T})$.

Definition 5.11. Suppose $M$ is a suitable $a$-premouse. We say $R$ is a pseudonormal iterate of $M$ if, and only if, $R$ is suitable and there is a normal iteration tree $\mathcal{T}$ on $M$ such that either $\mathcal{T}$ has successor length and $R$ is the last model of $\mathcal{T}$ or $\mathcal{T}$ is maximal and $R=L p^{\omega}(\mathcal{M}(\mathcal{T}))$.

As usual, this notion can easily be generalized to stacks of normal trees, but we omit the technical details. The interested reader can find them in a different setting for example in [37], [25], or [20]. The following lemmas are the analogues of Theorems 3.14 and 3.16 in [37]. The proofs are similar to the ones in [37] and [29] and use absoluteness as in the proof of Lemma 5.6; we omit further details.

Lemma 5.12 (Pseudocomparison). Suppose $M$ and $N$ are countable short tree iterable, suitable a-premice. Then, they have a common pseudonormal iterate $R$ such that $R \in L[T, z]$, where $z$ is a real coding $M$ and $N$. Moreover, $\delta_{R} \leq$ $\left(\max \left\{\delta_{M}, \delta_{N}\right\}^{+}\right)^{L[T, z]}=\omega_{1}^{L[T, z]}$.

Lemma 5.13 (Pseudogenericity iteration). Let $M$ be a countable, short tree iterable, suitable a-premouse. Then for a cone of reals $z, M$ is countable in $L[T, z]$, and there is a nondropping pseudonormal iterate $R$ of $M$ in $L[T, z]$ such that $z$ is generic over $R$ for Woodin's extender algebra at $\delta_{R}$, and $\delta_{R}=\omega_{1}^{L[T, z]}$.

5.2. The models $\mathcal{R}_{a}^{x}$. As before, let $a$ be an arbitrary countable transitive set. Let $x$ be a real such that $a \in L[x]$. We consider the simultaneous pseudocomparison of all short tree iterable, suitable $a$-premice coded by some real $z \leq_{T} x$. We carry out this pseudocomparison while at the same time performing a pseudogenericity iteration making every $z \leq_{T} x$ generic over the common part of the final model. Note that there are only countably many reals $z \leq_{T} x$ for every fixed real $x$ and let $\mathcal{R}^{-}=\mathcal{R}_{a}^{x,-}$ denote the resulting model. That is, either $\mathcal{R}^{-}$is the common last model of the iteration trees obtained by the process described above, or all of these trees are maximal and $\mathcal{R}^{-}$is the common part model of one (and hence all) of these trees. Moreover, let $\mathcal{R}=\mathcal{R}_{a}^{x}=L p\left(\mathcal{R}_{a}^{x,-}\right)$ and $\delta=\mathcal{R}_{a}^{x,-} \cap$ Ord.

LEMMA 5.14. We have the following properties.

(1) As an $\mathcal{R}^{-}$-premouse, no level of $\mathcal{R}$ projects across $\delta$,

(2) $\delta_{\mathcal{R}}=\delta$ is a Woodin cardinal in $\mathcal{R}$,

(3) $\mathcal{P}(\delta) \cap \mathcal{R}=\mathcal{P}(\delta) \cap \mathrm{OD}_{\mathcal{R}^{-} \cup\left\{\mathcal{R}^{-}\right\}}$,

(4) $\mathcal{P}(a) \cap \mathcal{R}=\mathcal{P}(a) \cap \mathrm{OD}_{a \cup\{a\}}$, and

(5) $\omega_{1}^{L[T, x]}=\delta$.

Proof. We carry out the pseudocomparisons and pseudogenericity iterations to obtain $\mathcal{R}^{-}$within $L[T, x]$ in the sense of Lemmas 5.12 and 5.13.

Claim 5.15. The pseudocomparisons reach a limit stage in which all of the iteration trees are maximal.

PROOF. If the pseudocomparisons reach a limit stage in which one iteration tree $\mathcal{T}$ is maximal, this already implies that all iteration trees are maximal since they agree on their common part model and thus a short iteration tree $\mathcal{U}$ would provide a $\mathcal{Q}$-structure $\mathcal{Q}(\mathcal{U}) \unlhd L p(\mathcal{M}(\mathcal{U}))=L p(\mathcal{M}(\mathcal{T}))$ for $\mathcal{T}$, contradicting the maximality of $\mathcal{T}$. 
Therefore, we can suppose toward a contradiction that all iteration trees occurring in the pseudocomparisons are short. Then the pseudocomparisons are in fact comparisons and they end successfully using the short tree iteration strategies. They give rise to a last model $\mathcal{R}^{*}$ such that every $z \leq_{T} x$ is generic over $\mathcal{R}^{*}$ for Woodin's extender algebra. Moreover, the main branches through all iteration trees in the comparisons are nondropping and we have elementary iteration embeddings

$$
j_{\mathcal{N}}: \mathcal{N} \rightarrow \mathcal{R}^{*}
$$

for each short tree iterable, suitable $a$-premouse $\mathcal{N}$ coded by some real $z \leq_{T} x$. In particular, $\mathcal{R}^{*}$ is suitable, witnessed by a Woodin cardinal $\delta^{*}=\delta_{\mathcal{R}^{*}}$.

The proof of the comparison lemma (cf. e.g., the claim in the proof of [35, Theorem 3.11]) shows that, if a coiteration terminates successfully, the comparison process lasts at most countably many steps in $L[T, x]$, and so $\mathcal{R}^{*}$ is countable in $L[T, x]$. By construction, $x$ is generic over $\mathcal{R}^{*}$ for Woodin's extender algebra at $\delta^{*}$, so we shall write $\mathcal{R}^{*}[x]$ for the corresponding generic extension.

SubClaim 5.16. $\mathbb{R} \cap L[T, x] \subseteq \mathbb{R} \cap \mathcal{R}^{*}[x]$.

Proof. Recall that Woodin's extender algebra at $\delta^{*}$ has the $\delta^{*}$-c.c. and hence $\left(\left(\delta^{*}\right)^{+}\right)^{\mathcal{R}^{*}}=\left(\left(\delta^{*}\right)^{+}\right)^{\mathcal{R}^{*}[x]}$. Let $\gamma=\left(\left(\delta^{*}\right)^{+}\right)^{\mathcal{R}^{*}}$. Consider the countable set $\mathcal{R}^{*}[x] \mid \gamma$ and the model $L\left[T, \mathcal{R}^{*}[x] \mid \gamma\right]$. Since $x \in \mathcal{R}^{*}[x] \mid \gamma$, we have that $L[T, x] \subseteq$ $L\left[T, \mathcal{R}^{*}[x] \mid \gamma\right]$. Now, Lemma 5.2 implies that every real $y$ in $L\left[T, \mathcal{R}^{*}[x] \mid \gamma\right]$, belongs to an $\omega_{1}$-iterable $\mathcal{R}^{*}[x] \mid \gamma$-premouse $N^{y}$. By taking an initial segment if necessary, we can assume that $N^{y}$ is sound and $\rho_{\omega}\left(N^{y}\right) \leq \gamma$. Since $L[T, x] \subseteq L\left[T, \mathcal{R}^{*}[x] \mid \gamma\right]$, it suffices to show that every real $y$ in an $\omega_{1}$-iterable $\mathcal{R}^{*}[x] \mid \gamma$-premouse $N^{y}$ belongs to $\mathcal{R}^{*}[x]$.

Let $\bar{N}^{y}=\mathcal{P}^{N^{y}}\left(\mathcal{R}^{*} \mid \gamma\right)$ be the $\mathcal{R}^{*} \mid \gamma$-premouse obtained as the result of a $\mathcal{P}$ construction above $\mathcal{R}^{*} \mid \gamma$ inside $N^{y}$ in the sense of [27] or [33, Section 3]. As the size of the extender algebra at $\delta^{*}$ is small, $\bar{N}^{y}$ is again a premouse and by definability of the forcing $\rho_{\omega}\left(\bar{N}^{y}\right) \leq \gamma$ (see, for example [33, Section 3] for a similar argument). Therefore, the suitability of $\mathcal{R}^{*}$ yields $\bar{N}^{y} \unlhd \mathcal{R}^{*}$ and hence $N^{y}=\bar{N}^{y}[x] \unlhd \mathcal{R}^{*}[x]$, where $\bar{N}^{y}[x]$ and $\mathcal{R}^{*}[x]$ are construed as $\mathcal{R}^{*}[x] \mid \gamma$-premice. Thus $y \in \mathcal{R}^{*}[x]$, as desired.

Since $\mathcal{R}^{*}$ is countable in $L[T, x]$, in particular $\delta^{*}$, the Woodin cardinal in $\mathcal{R}^{*}$, is countable in $L[T, x]$. Using the subclaim, this implies that $\delta^{*}$ is countable in $\mathcal{R}^{*}[x]$. But the extender algebra at $\delta^{*}$ in $\mathcal{R}^{*}$ has the $\delta^{*}$-c.c., so $\delta^{*}$ remains a cardinal in $\mathcal{R}^{*}[x]$ - a contradiction.

Since all iteration trees are maximal, we have $\mathcal{R}^{-}=\mathcal{M}(\mathcal{T})$, where $\mathcal{T}$ is an iteration tree of limit length on a suitable $a$-premouse coded by some real $z \leq_{T} x$. Then $\mathcal{R}=L p\left(\mathcal{R}^{-}\right)$satisfies (1) and (2).

For (3), note that $\mathcal{P}(\delta) \cap \mathrm{OD}_{\mathcal{R}^{-} \cup\left\{\mathcal{R}^{-}\right\}}$is the set of all subsets of $\delta$ which belong to an $\omega_{1}$-iterable $\mathcal{R}^{-}$-premouse. Since $\mathcal{R}=L p\left(\mathcal{R}^{-}\right)$these correspond to initial segments of $\mathcal{R}$.

This also implies that every subset of $a$ in $\mathrm{OD}_{a \cup\{a\}}$ belongs to $\mathcal{R}$ since $\mathrm{OD}_{a \cup\{a\}} \subseteq$ $\mathrm{OD}_{\mathcal{R}^{-} \cup\left\{\mathcal{R}^{-}\right\}}$. For the other inclusion in (4), suppose that $b$ is a subset of $a$ and $b \in \mathcal{R}$. As no new subsets of $a$ are added during the iteration, $b \in N$ for some short tree iterable, suitable $a$-premouse $N$ coded by some real $z \leq_{T} x$. But then 
$N \mid \eta$ is $\omega_{1}$-iterable for every ordinal $\eta$ such that $\rho_{\omega}(N \mid \eta)=a$ as, in these cases, $\mathcal{Q}$-structures exist. Hence, $b$ belongs to an $\omega_{1}$-iterable $a$-premouse and hence to $\mathrm{OD}_{a \cup\{a\}}$.

Finally, (5) follows by the same argument as in the proof of the subclaim since the assumption that $\delta<\omega_{1}^{L[T, x]}$ together with (1) and (2) suffices to derive the contradiction.

The construction of $\mathcal{R}_{a}^{x}$ depends only on the Turing degree of $x$, i.e., $x \equiv_{T} y$ implies $\mathcal{R}_{a}^{x}=\mathcal{R}_{a}^{y}$. Therefore, we will also write $\mathcal{R}_{a}^{d}$ for $\mathcal{R}_{a}^{x}$, if $d=[x]_{T}$.

5.3. Prikry-like forcing a premouse. We define a Prikry-like partial order $\mathbb{P}$ to add a premouse with infinitely many Woodin cardinals. Let $\mathcal{D}$ denote the set of all Turing degrees and let $\mu$ denote the Martin measure on $\mathcal{D}$. Further, let $\mathcal{D}^{m}$ be the set of all increasing sequences of Turing degrees of length $m$ and $\mu_{m}$ be the measure on $\mathcal{D}^{m}$ induced by the product of $\mu$. More precisely, let $\mu_{0}=\mu$ and assume inductively that $\mu_{k}$ is already defined on $\mathcal{D}^{k}$ for some $k<m$. Then we let for any $X \in \mathcal{D}^{k+1}$, $\mu_{k+1}(X)=1$ if, and only if, for $\mu_{0}$-a.e. $d_{0}$ and $\mu_{k}$-a.e. $\left(d_{1}, \ldots, d_{k}\right),\left(d_{0}, \ldots, d_{k}\right) \in X$.

To define the Prikry-like partial order $\mathbb{P}$, we first define a sequence of premice along an increasing sequence of Turing degrees. For $\vec{d}=\left(d_{0}, \ldots, d_{m}\right) \in \mathcal{D}^{m+1}$, we let

$$
\mathcal{Q}_{0}^{\vec{d}}(a)=\mathcal{R}_{a}^{d_{0}}
$$

if $d_{0}$ is large enough so that $\mathcal{R}_{a}^{d_{0}}$ is defined, and recursively

$$
\mathcal{Q}_{i+1}^{\vec{d}}(a)=\mathcal{R}_{\mathcal{Q}_{i}^{d}(a)}^{d_{i+1}}
$$

for $i<m$, if $d_{i+1}$ is large enough so that $\mathcal{R}_{\mathcal{Q}_{i}^{d}(a)}^{d_{i+1}}$ is defined.

Recall that $U$ is a $\Sigma_{1}^{2}$ set which is universal for $\Sigma_{1}^{2}$ in $V=M_{1}(A)$. Now, the conditions in $\mathbb{P}$ are of the form $(s, \vec{X})$, where

1. $s=\left(\mathcal{S}_{0}, \ldots, \mathcal{S}_{n}\right)$ is a sequence of premice such that for some $\overrightarrow{d_{s}} \in \mathcal{D}^{n+1}$, $\mathcal{S}_{i}=\mathcal{Q}_{i}^{\overrightarrow{d_{s}}}(\emptyset)$ for all $i \leq n$; and

2. $\vec{X}=\left(X_{k}: k<\omega\right) \in L(U, A)^{7}$ is a sequence of sets such that for all $k<\omega$,

(a) $X_{k}$ is a collection of $(k+1)$-sequences of premice, and

(b) $\left(\mathcal{Q}_{0}^{\vec{d}}\left(\mathcal{S}_{n}\right), \ldots, \mathcal{Q}_{k}^{\vec{d}}\left(\mathcal{S}_{n}\right)\right) \in X_{k}$ for $\mu_{k+1}$-a.e. $\vec{d} \in \mathcal{D}^{k+1}$.

We call $s$ the stem of the condition $(s, \vec{X})$. For two conditions $(s, \vec{X})$ and $(r, \vec{Y})$ in $\mathbb{P}$ with $s=\left(\mathcal{S}_{0}, \ldots, \mathcal{S}_{n}\right)$ and $r=\left(\mathcal{R}_{0}, \ldots, \mathcal{R}_{m}\right)$, we let $(s, \vec{X}) \leq(r, \vec{Y})$ if, and only if, one of the following holds:

1. $s=r$ and $X_{i} \subseteq Y_{i}$ for all $i<\omega$; or

2. for some $k<\omega$ and a sequence $\left(\mathcal{Q}_{0}^{\vec{d}}\left(\mathcal{R}_{m}\right), \ldots, \mathcal{Q}_{k}^{\vec{d}}\left(\mathcal{R}_{m}\right)\right) \in Y_{k}$ given by some $\vec{d} \in \mathcal{D}^{k+1}$

(a) $s=r \succ\left(\mathcal{Q}_{0}^{\vec{d}}\left(\mathcal{R}_{m}\right), \ldots, \mathcal{Q}_{k}^{\vec{d}}\left(\mathcal{R}_{m}\right)\right)$, and

(b) for all $i<\omega$ and sequences $\vec{e} \in \mathcal{D}^{i+1}$ such that $\left(\mathcal{Q}_{0}^{\vec{e}}\left(\mathcal{S}_{n}\right), \ldots, \mathcal{Q}_{i}^{\vec{e}}\left(\mathcal{S}_{n}\right)\right)$ is defined and belongs to $X_{i}$, we have

$$
\left(\mathcal{Q}_{0}^{\vec{d} \vec{e}}\left(\mathcal{R}_{m}\right), \ldots, \mathcal{Q}_{k}^{\vec{d} \vec{e}}\left(\mathcal{R}_{m}\right), \mathcal{Q}_{k+1}^{\vec{d} \vec{e}}\left(\mathcal{R}_{m}\right), \ldots, \mathcal{Q}_{k+i+1}^{\vec{d} \vec{e}}\left(\mathcal{R}_{m}\right)\right) \in Y_{k+i+1}
$$

\footnotetext{
${ }^{7}$ The reason for requiring $\vec{X} \in L(U, A)$ will become apparent in the proof of Lemma 5.23.
} 
The next lemma shows that $\mathbb{P}$ has the Prikry property. As the proof is analogous to e.g., the proof of Corollary 6.39 in [37], we omit it.

LEMMA 5.17. Let $(s, \vec{X}) \in \mathbb{P}$ be a condition and $\Lambda$ a countable set of sentences in the forcing language. Then there is some $(s, \vec{Y}) \leq(s, \vec{X})$ such that $(s, \vec{Y})$ decides $\phi$, for all $\phi \in \Lambda$.

Now fix a $G$ which is $\mathbb{P}$-generic over $M_{1}(A)$ and let $\overrightarrow{\mathcal{Q}}=\left(\mathcal{Q}_{n}: n<\omega\right)$ be the union of the stems of conditions in $G$. Write $\delta_{n}$ for the largest cardinal in $\mathcal{Q}_{n}$. By definition, all $\mathcal{Q}_{n}$ are such that $\mathcal{Q}_{n}=L p\left(\mathcal{Q}_{n} \mid \delta_{n}\right)$ and $L p^{\omega}\left(\mathcal{Q}_{n} \mid \delta_{n}\right)$ is a suitable premouse, so $\delta_{n}$ is a Woodin cardinal in $\mathcal{Q}_{n}$. Let

$$
\mathcal{Q}_{\infty}=\bigcup_{n<\omega} \mathcal{Q}_{n} .
$$

LEMMA 5.18. The following hold:

(1) for all $n<\omega, \mathcal{P}\left(\delta_{n}\right) \cap L[\overrightarrow{\mathcal{Q}}] \subseteq \mathcal{Q}_{n}$,

(2) for all $n<\omega, \delta_{n}$ is a Woodin cardinal in $L[\overrightarrow{\mathcal{Q}}]$,

(3) $\mathcal{Q}_{n}=\mathcal{Q}_{\infty} \mid\left(\delta_{n}^{+}\right)^{\mathcal{Q}_{\infty}}$; hence, $L\left[\mathcal{Q}_{\infty}\right]=L[\overrightarrow{\mathcal{Q}}]$.

Proof. We show (1), from which (2) and (3) follow. Let us first show that $\mathcal{P}\left(\delta_{n}\right) \cap$ $\mathcal{Q}_{n+1} \subseteq \mathcal{Q}_{n}$; a consequence of this is that $\mathcal{P}\left(\delta_{n}\right) \cap \mathcal{Q}_{m} \subseteq \mathcal{Q}_{n}$ whenever $n<m$. To see this, suppose there is a subset $a$ of $\delta_{n}$ which is in $\mathcal{Q}_{n+1}$. By Lemma 5.14(4), $\mathcal{P}\left(\delta_{n}\right) \cap \mathcal{Q}_{n+1}=\mathcal{P}\left(\delta_{n}\right) \cap \mathrm{OD}_{\mathcal{Q}_{n} \cup\left\{\mathcal{Q}_{n}\right\}}$. Lemma 5.14(3) implies that $\mathcal{P}\left(\delta_{n}\right) \cap \mathcal{Q}_{n}=$ $\mathcal{P}\left(\delta_{n}\right) \cap \mathrm{OD}_{\mathcal{Q}_{n}^{-} \cup\left\{\mathcal{Q}_{n}^{-}\right\}}$, but this is equal to $\mathcal{P}\left(\delta_{n}\right) \cap \mathrm{OD}_{\mathcal{Q}_{n} \cup\left\{\mathcal{Q}_{n}\right\}}$ by definability of $\mathcal{Q}_{n}=L p\left(\mathcal{Q}_{n}^{-}\right)$. Hence, we have $a \in \mathcal{Q}_{n}$, as desired.

To prove (1), let $a \in \mathcal{P}\left(\delta_{n}\right) \cap L[\overrightarrow{\mathcal{Q}}]$. Let $\dot{a}$ be a term defining $a$ from $\overrightarrow{\mathcal{Q}}$ and an ordinal parameter in $M_{1}(A)$ [G]. The Prikry property (Lemma 5.17) yields a $k<\omega$ and a condition $(s, \vec{X})$ with $s$ of the form $\left(s_{0}, \ldots, s_{k}\right)$, with $n<k$, which decides all statements of the form " $\xi \in \dot{a}$ ". By genericity we can choose $(s, \vec{X}) \in G$, so that, in particular, $s_{i}=\mathcal{Q}_{i}$ for all $i \leq k$.

Claim 5.19. We have $\xi \in a$ if, and only if,

$\exists t \exists \vec{Y}\left(t\right.$ is of the form $\left(t_{0}, \ldots, t_{k}\right) \wedge t_{i}=\mathcal{Q}_{i}$ for all $\left.i \leq k \wedge(t, \vec{Y}) \Vdash \xi \in \dot{a}\right)$.

Proof. If $\xi \in a$, then the condition $(s, \vec{X})$ is a witness for the displayed equation. Conversely, suppose there is some condition $(t, \vec{Y})$ as in the displayed equation, but $\xi \notin a$. Then we must have $(s, \vec{X}) \Vdash \xi \notin \dot{a}$. Note that $t=s$. Define $\vec{Z}$ by $Z_{i}=X_{i} \cap Y_{i}$ for all $i<\omega$. Then $(s, \vec{Z}) \leq(s, \vec{X})$ and $(s, \vec{Z})=(t, \vec{Z}) \leq(t, \vec{Y})$. Now, let $H$ be $\mathbb{P}$-generic over $M_{1}(A)$ such that $H$ contains $(s, \vec{Z})$. Then, in $M_{1}(A)[H]$, both $\xi \in a$ and $\xi \notin a$ hold, a contradiction.

The claim yields that $a \in \mathrm{OD}_{\mathcal{Q}_{k} \cup\left\{\mathcal{Q}_{k}\right\}}$ and thus $a \in \mathrm{OD}_{\mathcal{Q}_{k}^{-} \cup\left\{\mathcal{Q}_{k}^{-}\right\}} \cap \mathcal{P}\left(\delta_{k}\right)=$ $\mathcal{Q}_{k} \cap \mathcal{P}\left(\delta_{k}\right)$, by Lemma 5.14(3). So in particular $a \in \mathcal{Q}_{k} \cap \mathcal{P}\left(\delta_{n}\right)$. By the remark at the beginning of the proof, this implies $a \in \mathcal{Q}_{n}$, as desired.

Write $\lambda=\sup _{n<\omega} \delta_{n}$ and fix any premouse $\mathcal{P} \models$ ZFC extending $\mathcal{Q}_{\infty}$ in which $\lambda$ remains a cardinal. We form a derived model of $\mathcal{P}$. More precisely, working in $M_{1}(A)[G]$, let $\mathbb{S}$ be the partial order consisting of sequences $\left(h_{0}, \ldots, h_{k}\right)$ such that for all $0 \leq n \leq k, h_{n} \in M_{1}(A)$ is $\operatorname{Col}\left(\omega, \delta_{n}\right)$-generic over $\mathcal{Q}_{n}$. The order on $\mathbb{S}$ is sequence extension. Fix $\hat{h} \mathbb{S}$-generic over $M_{1}(A)[G]$, let $\left(h_{n}: n<\omega\right)$ be the induced sequence, and let $h$ be given by $h(n, m)=h_{n}(m)$. We may abuse notation 
and identify $\hat{h}$ with $h$ and with the corresponding $\operatorname{Col}(\omega,<\lambda)$-generic filter over $M_{1}(A)[G]$, since $\left(\delta_{n}: n<\omega\right)$ is definable from $\mathcal{Q}_{\infty}$ by the previous lemma.

Using this $h$, we can build the derived model of $\mathcal{P}$ : Write

$$
\mathbb{R}_{h}^{*}=\bigcup_{n \in \omega} \mathbb{R} \cap \mathcal{P}\left[h\left\lceil\delta_{n}\right]\right.
$$

and

$$
\begin{aligned}
\operatorname{Hom}_{h}^{*}=\{p[S] & \cap \mathbb{R}_{h}^{*} \mid \exists n<\omega\left(\mathcal { P } \left[h\left\lceil\delta_{n}\right] \vDash S\right.\right. \text { is a } \\
& <\lambda \text {-absolutely complemented tree })\} .
\end{aligned}
$$

Note that $\mathbb{R}_{h}^{*}$, and $\mathrm{Hom}_{h}^{*}$ only depend on $\mathcal{Q}_{\infty}$ and $h$, not on the full premouse $\mathcal{P}$, so this notation makes sense. For this reason, we sometimes do not distinguish between $\mathcal{P}$ and $\mathcal{Q}_{\infty}$ in what follows.

5.4. Preparation for adding extenders on top. Our next goal is to add the extenders of $M_{1}(A)$ on top of $\mathcal{Q}_{\infty}$ while preserving the Woodin cardinals of $\mathcal{Q}_{\infty}$ to obtain a model with $\omega+1$ Woodin cardinals. This will be done via a $\mathcal{P}$-construction. For this, some preparation is needed - we need to show e.g.,

$$
L_{\xi}\left[\mathcal{Q}_{\infty}\right][h]=\left(M_{1}(A) \mid \xi\right)[G][h]
$$

for some ordinal $\xi$ below the Woodin cardinal of $M_{1}(A)$.

We first need the following lemmas which again essentially can be found also in [31]. Recall that we have $V=M_{1}(A)$ and write $\mathbb{R}^{V}=M_{1}(A) \cap \mathbb{R}=A$.

LEMMA 5.20. $\mathbb{R}_{h}^{*}=\mathbb{R}^{V}$.

Proof. $\mathbb{R}_{h}^{*} \subseteq \mathbb{R}^{V}$ is easy to see as each pair $\left(\mathcal{Q}_{n}, h_{n}\right)$ is in $M_{1}(A)$, so let $x \in \mathbb{R}^{V}$ for the other inclusion. Let $(s, \vec{X}) \in \mathbb{P}$ be an arbitrary condition in the Prikry-like forcing defined above, say $s=\left(\mathcal{S}_{0}, \ldots, \mathcal{S}_{k}\right)$. For each $n<\omega$ consider

$$
Y_{n}=\left\{t \in X_{n} \mid \exists \vec{d} \in \mathcal{D}^{n+1}\left(x \leq_{T} d_{0} \wedge t=\left(\mathcal{Q}_{0}^{\vec{d}}\left(\mathcal{S}_{k}\right), \ldots, \mathcal{Q}_{n}^{\vec{d}}\left(\mathcal{S}_{k}\right)\right)\right)\right\}
$$

and note that $(s, \vec{Y}) \leq(s, \vec{X})$. Moreover, since pseudogenericity iterations are included in the construction of the premice $\mathcal{R}_{a}^{d}$, it follows by density that for all $i>k, x$ is generic over $\mathcal{Q}_{i}$ for Woodin's extender algebra at $\delta_{i}$. Hence $x \in \mathbb{R}_{h}^{*}$.

LEMMA 5.21. $\operatorname{Hom}_{h}^{*}=\Delta_{1}^{2}$.

Proof. We first show $\Delta_{1}^{2} \subseteq \operatorname{Hom}_{h}^{*}$. Let $B \in \Delta_{1}^{2}$, so that $B$ is, since $\Sigma_{1}^{2}$ has the scale property, Suslin and co-Suslin. More precisely, choose $z \in \mathbb{R}$ such that $B \in \Delta_{1}^{2}(z)$ and choose $i \in \omega$ large enough so that $z \in \mathcal{Q}_{i}\left[h_{i}\right]$ (this exists by the previous lemma). From $T$ and $z$, one can construct trees $S_{0}$ and $S_{1}$ on $\omega \times \kappa$, for some ordinal $\kappa<\delta_{1}^{2}$, such that $S_{0}$ and $S_{1}$ project to $B$ and $\mathbb{R} \backslash B$, respectively. We have

$$
S_{0}, S_{1} \in L\left[T, \mathcal{Q}_{j}, h_{i}\right]
$$

for every $j \geq i$. Moreover, by the absoluteness of well-foundedness, if $x \in$ $L\left[T, \mathcal{Q}_{j}, h_{i}\right] \cap \mathbb{R}$, then

$$
L\left[T, \mathcal{Q}_{j}, h_{i}\right] \models x \in p\left[S_{0}\right] \cup p\left[S_{1}\right] .
$$

Moreover, if $\mathbb{Q}$ is a small partial order in $L\left[T, \mathcal{Q}_{j}, h_{i}\right]$ (say, of size strictly less than $\left.\delta_{j}\right)$ and $g$ is $\mathbb{Q}$-generic over $L\left[T, \mathcal{Q}_{j}, h_{i}\right]$, then

$$
L\left[T, \mathcal{Q}_{j}, h_{i}\right][g] \models \mathbb{R}=p\left[S_{0}\right] \cup p\left[S_{1}\right],
$$


for (since $\delta_{j}$ is countable in $V$ ) otherwise there is a $\mathbb{Q}$-generic $g$ over $L\left[T, \mathcal{Q}_{j}, h_{i}\right]$ with $g \in V$ such that

$$
L\left[T, \mathcal{Q}_{j}, h_{i}\right][g] \models \exists x \in \mathbb{R}\left(x \notin p\left[S_{0}\right] \cup p\left[S_{1}\right]\right) .
$$

However, such an $x$ does belong to one of $p\left[S_{0}\right]$ or $p\left[S_{1}\right]$ in $V$ and thus it must do too in $L\left[T, \mathcal{Q}_{j}, h_{i}\right][g]$ (by the absoluteness of well-foundedness).

We have shown that in $L\left[T, \mathcal{Q}_{j}, h_{i}\right]$ there are $<\delta_{j}$-absolutely complementing trees $S_{0}$ and $S_{1}$ that project to $B \cap L\left[T, \mathcal{Q}_{j}, h_{i}\right]$ and $(\mathbb{R} \backslash B) \cap L\left[T, \mathcal{Q}_{j}, h_{i}\right]$, respectively.

The trees $S_{0}$ and $S_{1}$ might be big (in principle $\kappa$ might be of arbitrarily large size below $\delta_{1}^{2}$ ). Let $M$ be an elementary substructure of some large $L_{\alpha}\left[T, \mathcal{Q}_{j}, h_{i}\right]$ such that

1. $M \in L\left[T, \mathcal{Q}_{j}, h_{i}\right]$,

2. $L\left[T, \mathcal{Q}_{j}, h_{i}\right] \models|M|=\delta_{j}$,

3. $L_{\delta_{j}}\left[T, \mathcal{Q}_{j}, h_{i}\right] \subseteq M$, and

4. $S_{0}, S_{1} \in M$.

Let $S_{0}^{j}$ and $S_{1}^{j}$ be the images of $S_{0}$ and $S_{1}$ under the collapse embedding for $M$. Then, $S_{0}^{j}$ and $S_{1}^{j}$ are also $<\delta_{j}$-absolutely complementing and $S_{0}^{j}$ projects to $B \cap L\left[T, \mathcal{Q}_{j}, h_{i}\right][g]$ whenever $g$ is $\mathbb{Q}$-generic over $L\left[T, \mathcal{Q}_{j}, h_{i}\right]$ for a partial order $\mathbb{Q}$ in $L\left[T, \mathcal{Q}_{j}, h_{i}\right]$ of size strictly less than $\delta_{j}$.

Claim 5.22. For all $j>i, S_{0}^{j}, S_{1}^{j} \in \mathcal{Q}_{\infty}\left[h_{i}\right]$.

Proof. Note that $h_{i}$ is $\operatorname{Col}\left(\omega, \delta_{i}\right)$-generic over $\mathcal{Q}_{j+1}$. Recall that by definition, $\mathcal{Q}_{j+1}=\mathcal{R}_{\mathcal{Q}_{j}}^{d}$ for some Turing degree $d$. Therefore we have, for such a $d$, by an argument similar to that in the proof of Subclaim 5.16 that

$$
\mathcal{Q}_{j+1}\left[h_{i}\right]=\mathcal{R}_{\mathcal{Q}_{j}\left[h_{i}\right]}^{d} .
$$

Now, by Lemma 5.2, every subset of $\mathcal{Q}_{j}$ in $L\left[T, \mathcal{Q}_{j}, h_{i}\right]$ is definable from $\mathcal{Q}_{j}$, $h_{i}$, and ordinal parameters in $V . S_{0}^{j}$ and $S_{1}^{j}$ are essentially subsets of $\delta_{j}$ in $L\left[T, \mathcal{Q}_{j}, h_{i}\right]$, so they are definable in $V$ from $\mathcal{Q}_{j}, h_{i}$, and ordinal parameters. By Lemma 5.14(4),

$$
\mathcal{P}\left(\mathcal{Q}_{j}\left[h_{i}\right]\right) \cap \mathcal{R}_{\mathcal{Q}_{j}\left[h_{i}\right]}^{d}=\mathcal{P}\left(\mathcal{Q}_{j}\left[h_{i}\right]\right) \cap \mathrm{OD}_{\mathcal{Q}_{j}\left[h_{i}\right] \cup\left\{\mathcal{Q}_{j}\left[h_{i}\right]\right\}} .
$$

This implies that $S_{0}^{j}$ and $S_{1}^{j}$ belong to $Q_{j+1}\left[h_{i}\right]$, which proves the claim.

It follows that $S_{0}^{j}$ and $S_{1}^{j}$ are $<\delta_{j}$-absolutely complementing trees in $\mathcal{Q}_{\infty}\left[h_{i}\right]$ since by an argument as in the proof of Lemma 5.18, for every $g$ which is generic for a partial order of size strictly less than $\delta_{j}, \mathcal{Q}_{\infty}[g] \cap \mathcal{P}\left(\delta_{j}\right) \subseteq \mathcal{Q}_{j}[g]$. In particular, $B \cap \mathcal{Q}_{\infty}\left[h_{i}\right]$ is $<\lambda$-universally Baire in $\mathcal{Q}_{\infty}\left[h_{i}\right]$. The canonical extension $B^{*}$ of $B \cap \mathcal{Q}_{\infty}\left[h_{i}\right]$ to a set in $H_{o m}^{*}$ is in fact unique and consistent with the trees $\left(S_{0}^{j}, S_{1}^{j}\right)$ for all $i<j<\omega$. Therefore $B=B^{*}$. This proves $\Delta_{1}^{2} \subseteq H_{h}$.

We now assume towards a contradiction that $\Delta_{1}^{2} \neq H_{h} m_{h}^{*}$, i.e., that the $\Delta_{1}^{2}$ sets form a proper Wadge initial segment of $\mathrm{Hom}_{h}^{*}$. Since Hom ${ }_{h}^{*}$ is closed under continuous reducibility and the $\Sigma_{1}^{2}$ set $U$ which is universal for $\Sigma_{1}^{2}$ is minimal in the Wadge hierarchy above the pointclass $\Delta_{1}^{2}$, it follows that $U \in H_{o m}^{*}$.

By definition of $\mathrm{Hom}_{h}^{*}$, there is some $i \in \omega$ and a $<\lambda$-absolutely complemented tree $S$ in $\mathcal{Q}_{\infty}\left[h_{i}\right]$ such that, since $\mathbb{R}^{V}=\mathbb{R}_{h}^{*}$ by Lemma 5.20, $p[S] \cap \mathbb{R}^{V}=\mathbb{R}^{V} \backslash U$. 
By Lemma 5.2, the relation

$$
x \notin \mathrm{OD}_{\{y\}}
$$

is $\Pi_{1}^{2}$, so it appears in a section of the complement of $U$. Using $S$, we can get a real $x$ such that $x \notin \mathrm{OD}_{\left\{\left(\mathcal{Q}_{i} \mid \delta_{i}, h_{i}\right)\right\}}$ but $x \in \mathcal{Q}_{\infty}\left[h_{i}\right]$. Then in fact $x \in \mathcal{Q}_{i}\left[h_{i}\right]$ by the argument in the proof of Lemma 5.18. However, $\mathcal{Q}_{i}=L p\left(\mathcal{Q}_{i} \mid \delta_{i}\right)$, so every real in $\mathcal{Q}_{i}\left[h_{i}\right]$ is definable from $\mathcal{Q}_{i} \mid \delta_{i}, h_{i}$, and ordinal parameters, which is a contradiction. This completes the proof of the lemma.

Recall that $\mathbb{R}^{V}=A$. Define $\xi_{0}$ to be the least $\xi>\Theta^{L(U, A)}$ such that $L_{\xi}(U, A) \vDash Z F$. Since $V \mid \xi_{0}$ is a countably iterable $A$-premouse, it is easy to see that

$$
V \mid \xi_{0}=L_{\xi_{0}}(U, A) .
$$

Finally, we have the following agreement between $L\left[\mathcal{Q}_{\infty}\right][h]$ and $L(U, A)[G][h]$ (as classes). Here we will use the extra condition " $\vec{X} \in L(U, A)$ " in the definition of the Prikry-like forcing $\mathbb{P}$ defined in Section 5.3 to ensure that $\mathbb{P} \in L(U, A)$.

Lemma 5.23. $L\left[\mathcal{Q}_{\infty}\right][h]=L(U, A)[G][h]$.

Proof. In $L\left[\mathcal{Q}_{\infty}\right][h]$, one can easily compute the derived model of $L\left[\mathcal{Q}_{\infty}\right]$ associated to $h$. Thus, using Lemma 5.21, it follows that $U \in L\left[\mathcal{Q}_{\infty}\right][h]$. By Lemma 5.20, $A=\mathbb{R}^{V} \in L\left[\mathcal{Q}_{\infty}\right][h]$ and, using $L(U, A)$ and $\mathcal{Q}_{\infty}$, one can easily define $\mathbb{P}$ and $G$. Conversely, from $G$ one can easily recover $\mathcal{Q}_{\infty}$.

This, together with the observation above, has the following corollary:

Corollary 5.24. $L_{\xi_{0}}\left[\mathcal{Q}_{\infty}\right][h]=V \mid \xi_{0}[G][h]$.

5.5. Adding extenders on top. Finally, we use a $\mathcal{P}$-construction (see, for example [33, Section 3] or [27]) to add extenders witnessing another Woodin cardinal on top of $\mathcal{Q}_{\infty}$. In Lemma 5.25, we first extend Corollary 5.24 to ordinals $\xi>\xi_{0}$ in order to obtain an appropriate background universe $W$ for the $\mathcal{P}$-construction. Using the fact that $V[G][h]$ is a forcing extension of the $A$-premouse $V=M_{1}(A)$ by a small forcing, the proof of this lemma is straightforward and similar to the argument in [33, Section 3], so we omit it.

LEMMA 5.25. There is a proper class $\left(\mathcal{Q}_{\infty}, h\right)$-premouse $W$ such that for any $\xi \geq \xi_{0}$,

1. $W \mid \xi$ has the same universe as $V \mid \xi[G][h]$,

2. for any $k<\omega, \rho_{k}(W \mid \xi)=\omega$ if, and only if, $\rho_{k}(V \mid \xi)=A$, and

3. for any $k<\omega$, if $\rho_{k}(W \mid \xi)>\omega$, then $\rho_{k}(W \mid \xi)=\rho_{k}(V \mid \xi)$ and $p_{k+1}(W \mid \xi)=$ $p_{k+1}(V \mid \xi)$.

Let $\mathcal{P}=\mathcal{P}^{W}\left(L_{\xi_{0}}\left[\mathcal{Q}_{\infty}\right]\right)$ be the result of a $\mathcal{P}$-construction above $L_{\xi_{0}}\left[\mathcal{Q}_{\infty}\right]$ performed inside $W$ and let $\mathcal{P}_{\xi}$ for $\xi \geq \xi_{0}$ denote the levels of the $\mathcal{P}$-construction. The following lemma shows that $\mathcal{P}$ is as desired.

LEMMA 5.26. The following hold:

(1) For $\xi \geq \xi_{0}$, if $X \subseteq \mathcal{P}_{\xi}$ is definable over $\mathcal{P}_{\xi}$ with parameters from $\mathcal{P}_{\xi}$, then $X \cap \mathcal{Q}_{\infty} \mid \delta_{n} \in \mathcal{Q}_{\infty}$ for all $n<\omega$.

(2) For all $\xi \geq \xi_{0}, \rho_{\omega}\left(\mathcal{P}_{\xi}\right) \geq \lambda$.

(3) $\mathcal{P}$ is a premouse and $\mathcal{P}[h]=W$.

(4) $\mathcal{P}$ has $\omega+1$ Woodin cardinals. 
Proof. For the proof of (1) note that $\mathcal{P}_{\xi}$ is definable over $V \mid \xi[G]$ from $\mathcal{Q}_{\infty}$ since the translation between $V \mid \xi[G][h]$ and $W \mid \xi$ is definable and $h$ is generic over $V \mid \xi[G]$ for a homogeneous forcing. Hence, if $X \subseteq \mathcal{P}_{\xi}$ is definable over $\mathcal{P}_{\xi}$ with parameters from $\mathcal{P}_{\xi}$, then $X \in V[G]$. We can assume without loss of generality that $X$ is a set of ordinals and let $\dot{X}_{n}$ for every $n<\omega$ be a term defining $X \cap \delta_{n}$ from $\mathcal{Q}_{\infty}$ and the ordinal parameter $\xi$. Now we can argue as in the proof of Lemma 5.18 to obtain $X \cap \delta_{n} \in \mathcal{Q}_{\infty}$, as desired.

Suppose (2) fails and let $\xi \geq \xi_{0}$ be least such that $\rho_{\omega}\left(\mathcal{P}_{\xi}\right)=\rho<\lambda$. Say this is witnessed by some set of ordinals $X \subseteq \rho$, which is definable over $\mathcal{P}_{\xi}$ with parameters from $\mathcal{P}_{\xi}$, but $X \notin \mathcal{P}_{\xi}$. Since $\lambda=\bigcup_{n<\omega} \delta_{n}$, there is some $n<\omega$ such that $\rho<\delta_{n}$. This means that $X=X \cap \delta_{n} \in \mathcal{Q}_{\infty}$ by (1). But $\mathcal{Q}_{\infty} \subseteq \mathcal{P}_{\xi}$, a contradiction.

Finally, (3) and (4) now follow from this and standard properties of the $\mathcal{P}$ construction, see, for example [27].

This finishes the proof of Theorem 1.1 and, by putting the active extender of $M_{1}^{\sharp}(A)$ on an initial of $\mathcal{P}$, e.g., as in [5, Section 2], also the proof of Theorem 5.1.

§6. Applications. We sketch a proof of the equiconsistencies of the schemata stated in Corollary 1.2.

Proof of Corollary 1.2. We start with the equiconsistency of (1), (2), and (3). Theorem 3.1 together with Theorem 4.1 immediately gives that the consistency of (1) implies the consistency of (2). Now suppose that (2) is consistent, say there is a model $M$ of ZF $+\mathrm{DC}+\mathrm{AD}$ with $n+5$ Woodin cardinals. Using a fully backgrounded extender construction as in [17], it is easy to see that $M_{n}^{\sharp}\left(\mathbb{R}^{M}\right)$ exists in $M$ and $M_{n}\left(\mathbb{R}^{M}\right) \cap \mathbb{R}=\mathbb{R}^{M}$. Moreover, as winning strategies for games of length $\omega$ on $\omega$ can be coded by reals, $M_{n}\left(\mathbb{R}^{M}\right) \models \mathrm{AD}$. Therefore the argument in Section 5 shows that there is a model of ZFC with $\omega+n$ Woodin cardinals. The direction from (3) to (1) follows from [23], where Neeman argues in Appendix A, using results of [15], that the existence of a model with $\omega+n$ Woodin cardinals and a measurable cardinal above them all implies the existence of a sufficiently iterable active premouse with $\omega+n$ Woodin cardinals to which Theorem 2A.3 in [23] applies.

Next, we argue that (4) is equiconsistent with (1) and (2). First, suppose that there is a model $V$ of ZF $+\mathrm{DC}+\mathrm{AD}$ and, say, $\boldsymbol{\Pi}_{n+5}^{1}$-determinacy for games of length $\omega$ on $\mathbb{R}$ and work in this model. The methods of Section 3 can be used to conclude from $\Pi_{n+5}^{1}$-determinacy for games of length $\omega$ on reals that $M_{n}^{\sharp}(\mathbb{R})$ exists and is countably iterable (see [2] for details). Since $M_{n}^{\sharp}(\mathbb{R})$ contains all the reals, it in particular contains codes for all winning strategies for games on natural numbers of length $\omega$ which exist in $V$. Therefore $M_{n}^{\sharp}(\mathbb{R}) \vDash$ AD witnesses (2). For the other direction, suppose there is model of ZFC in which, say, $\boldsymbol{\Pi}_{n+5}^{1}$-determinacy holds for games of length $\omega^{2}$ on $\omega$. By the results in Section 3, there a countable set of reals $A$ such that $M_{n+4}(A) \vDash " \mathbb{R}=A "+\mathrm{AD}$. Now work inside $V=M_{n+4}(A)$ and write $\mathbb{R}=A$. Note that $V$ is a model of ZF $+\mathrm{DC}$ (see e.g., [19]). Since $M_{n+3}^{\sharp}(\mathbb{R})$ exists in $V$, it follows from the proof of projective determinacy in [24] that $\mathbf{\Pi}_{n}^{1}$-determinacy holds for games of length $\omega$ on $\mathbb{R}$ (see [2] for details).

Finally, we argue that (5) is equiconsistent with the other schemata. Suppose there is model of ZFC in which, say, $\boldsymbol{\Pi}_{n+5}^{1}$-determinacy holds for games of length 
$\omega^{2}$ on $\omega$. Then again by the results in Section 3 there a countable set of reals $A$ such that $M_{n+4}(A) \vDash " \mathbb{R}=A$ " $+\mathrm{AD}$ and we can work in $V=M_{n+4}(A)$. As $V$ has sufficiently many Woodin cardinals, every $\operatorname{Col}(\omega, \mathbb{R})$-generic extension $V[g]$ is closed under $x \mapsto M_{n}^{\sharp}(x)$ for all reals $x$ and hence satisfies $\Pi_{n+1}^{1}$-determinacy for games of length $\omega$ on $\omega$. For the other direction we show the consistency of (4). Suppose that there is a model $V$ of ZF $+\mathrm{DC}+\mathrm{AD}$ such that, say, $\boldsymbol{\Pi}_{n+5}^{1}$-determinacy for games of length $\omega$ on $\omega$ holds in every $\operatorname{Col}(\omega, \mathbb{R})$-generic extension of $V[g]$ of $V$. As $\mathbb{R}^{V}$ is a countable set of reals in $V[g], M_{n+1}^{\sharp}\left(\mathbb{R}^{V}\right)$ exists in $V[g]$. By homogeneity of the forcing, this implies that in $V$ there is a set of reals coding a model with $n+1$ Woodin cardinals which contains $\mathbb{R}^{V}$. This suffices to run the argument in [24] mentioned above which yields $\boldsymbol{\Pi}_{n}^{1}$-determinacy for games of length $\omega$ on $\mathbb{R}$. $\dashv$

We finish this article by sketching the modifications which are needed to prove Theorems 1.3 and 1.4 .

Proof OF TheOREM 1.3. Instead of Theorem 3.1, we now aim to obtain a countable set of reals $A$ such that $M_{\omega}(A) \cap \mathbb{R}=A$ and $M_{\omega}(A) \vDash \mathrm{ZF}+\mathrm{AD}$. For this purpose, we replace 1-smallness in the definition of $\varphi$-witness (see Definition 3.3) by $\omega$-smallness. Moreover, we replace $\Pi_{2}^{1}$-iterability in the definition of the game $\mathcal{G}_{\varphi, \psi}$ (see Definition 3.6) by the notion of weak iterability (also called $\supset{ }^{\mathbb{R}} \Pi_{1}^{1}$-iterability) as in [35, Definition 7.7]. Note that determinacy of games of length $\omega^{2}$ with $\partial^{\mathbb{R}} \Pi_{1}^{1}$ payoff suffices to show that this game is determined. Furthermore, Theorem 7.10 in [35] suffices to carry out the comparison arguments used to prove Theorem 3.1. The proofs in Sections 4 and 5 straightforwardly generalize to this context.

Proof OF TheOREM 1.4. Instead of Theorem 3.1, we now aim to obtain a countable set of reals $A$ such that there is an $A$-premouse $M$ of class $S_{\alpha}$ such that $M \cap \mathbb{R}=A$ and $M \vDash \mathrm{ZF}+\mathrm{AD}$. For this purpose, we replace 1-smallness in the definition of $\varphi$-witness (see Definition 3.3) by not being of class $S_{\alpha}$. Moreover, we replace $\Pi_{2}^{1}$-iterability in the definition of the game $\mathcal{G}_{\varphi, \psi}$ (see Definition 3.6) by the notion of $\Pi_{\alpha}^{1}$-iterability as in [1, Definition 4.1]. Note that determinacy of games of length $\omega^{2}$ with $\sigma$-projective payoff suffices to show that this game is determined. Furthermore, Lemmas 2.19 and 4.3 in [1] suffice to carry out the comparison arguments used to prove Theorem 3.1. The proofs in Sections 4 and 5 straightforwardly generalize to this context.

Acknowledgments. The first-listed author was partially supported by FWO grant 63374 and FWF grants P 31063 and P 31955. The second-listed author, formerly known as Sandra Uhlenbrock, was partially supported by FWF grant number P 28157.

\section{REFERENCES}

[1] J. P. Aguilera, $\sigma$-Projective determinacy, submitted, 2018.

[2] J. P. Aguilera and S. Müller, Projective Games on the Reals, 2019. Preprint available at https://muellersandra.github.io/publications/.

[3] J. P. Aguilera, S. Müller, and P. Schlicht, Long games and $\sigma$-projective sets, submitted, 2018. Preprint available at https://muellersandra.github.io/publications/.

[4] H. M. Friedman, Higher set theory and mathematical practice. Annals of Mathematical Logic, vol. 2 (1971), no. 3, pp. 325-357. 
[5] G. Fuchs, I. Neeman, and R. Schindler, A Criterion for coarse iterability. Archive for Mathematical Logic, vol. 49 (2010), pp. 447-467.

[6] L. A. Harrington, Analytic determinacy and $0^{\#}$, this Journal, vol. 43 (1978), pp. 685-693.

[7] L. A. Harrington and A. S. Kechris, On the determinacy of games on ordinals. Annals of Mathematical Logic, vol. 20 (1981), pp. 109-154.

[8] A. KanAmori, The Higher Infinite: Large Cardinals in Set Theory from their Beginnings, Springer Monographs in Mathematics, Springer, 2008.

[9] A. S. KeChris, The Axiom of determinancy implies dependent choices in $L(R)$, this JourNAL, vol. 49 (1984), no. 1, pp. 161-173.

[10] A. S. Kechris and Y. N. Moschovakis, Notes on the theory of scales, Games, Scales and Suslin Cardinals, the Cabal Seminar, vol. $\boldsymbol{i}$ (A. S. Kechris, B. Löwe, and J. R. Steel, editors), Cambridge University Press, New York, 2008, pp. 28-74.

[11] A. Kechris and R. Solovay, On the relative consistency strength of determinacy hypotheses. Transactions of the American Mathematical Society, vol. 290 (1985), no. 1, pp. 179-211.

[12] D. A. Martin, Measurable cardinals and analytic games. Fundamenta Mathematicae, vol. 66 (1970), pp. 287-291.

[13] - Borel determinacy. Annals of Mathematics, vol. 102 (1975), no. 2, pp. 363-371.

[14] D. A. Martin and J. R. Steel, A proof of projective determinacy. Journal of the American Mathematical Society, vol. 2 (1989), pp. 71-125. $1-73$

[15] - Iteration trees. Journal of the American Mathematical Society, vol. 7 (1994), no. 1, pp.

[16] - The extent of scales in $L(\mathbb{R})$, Games, Scales, and Suslin Cardinals, the Cabal Seminar, vol. $\boldsymbol{i}$ (A. S. Kechris, B. Löwe, and J. R. Steel, editors), Cambridge University Press, New York, 2008, pp. $110-120$.

[17] W. J. Mitchell and J. R. Steel, Fine Structure and Iteration Trees, Lecture Notes in Logic, Springer-Verlag, Berlin, New York, 1994.

[18] Y. N. Moschovakis, Descriptive Set Theory, second ed., Mathematical Surveys and Monographs, vol. 155, AMS, Providence, RI, 2009.

[19] S. Müller, The axiom of determinacy implies dependent choice in mice. Mathematical Logic Quarterly, vol. 65 (2019), no. 3, pp. 370-375.

[20] S. Müller and G. SARgSyan, HOD in inner models with Woodin cardinals, submitted, 2018. Preprint available at https://muellersandra.github.io/publications/.

[21] S. Müller, R. Schindler, and W. H. Woodin, Mice with finitely many Woodin cardinals from optimal determinacy hypotheses, Journal of Mathematical Logic, 2019. https://doi.org/10.1142/S0219061319500132.

[22] I. Neeman, Optimal proofs of determinacy. The Bulletin of Symbolic Logic, vol. 1 (1995), no. 3, pp. 327-339.

[23] - The Determinacy of Long Games, De Gruyter Series in Logic and its Applications, Walter de Gruyter, Berlin, 2004.

$[24]-$ Determinacy in L $(\mathbb{R})$, Handbook of Set Theory (M. Foreman and A. Kanamori, editors), Springer, Dordrecht, 2010, pp. 1877-1950.

[25] G. SARgSYAn, On the prewellorderings associated with directed systems of mice, this JournaL, vol. 78 (2013), no. 3, pp. 735-763.

[26] G. SARgsyan and J. Steel, The mouse set conjecture for sets of reals, this Journal, vol. 80 (2015), no. 2, p. 671-683.

[27] R. Schindler and J. R. Steel, The self-iterability of $L[E]$, this Journal, vol. 74 (2009), no. 3 , pp. 751-779.

[28] R. Schindler, J. R. Steel, and M. Zeman, Deconstructing inner model theory, this Journal, vol. 67 (2002), pp. 721-736.

[29] F. Schlutzenberg and N. D. Trang, Scales in hybrid mice over $\mathbb{R}$, submitted, 2016.

[30] J. R. Steel, Projectively well-ordered inner models. Annals of Pure and Applied Logic, vol. 74 (1995), pp. 77-104.

[31] - An optimal consistency strength lower bound for $\mathrm{AD}_{\mathbb{R}}$, unpublished, 2008.

[32] - Derived models associated to mice, Computational Prospects of Infinity - Part $\boldsymbol{i}$ (C.-T. Chong, editor), Lecture Notes Series, vol. 14, World Scientific, Singapore, 2008, pp. 105-193. 
[33] - Scales in $K(\mathbb{R})$, Games, Scales and Suslin Cardinals, the Cabal Seminar, vol. I (A. S. Kechris, B. Löwe, and J. R. Steel, editors), Cambridge University Press, New York, 2008, pp. 176-208. [34] - Scales in $L(\mathbb{R})$, Games, Scales and Suslin Cardinals, the Cabal Seminar, vol. I (A. S. Kechris, B. Löwe, and J. R. Steel, editors), Cambridge University Press, New York, 2008, pp. 130-175.

[35] —, An outline of inner model theory, Handbook of Set Theory (M. Foreman and A. Kanamori, editors), Springer, Dordrecht, 2010, pp. 1595-1684.

[36] —, A theorem of Woodin on mouse sets, Ordinal Definability and Recursion Theory, the Cabal Seminar, vol. III (A. S. Kechris, B. Löwe, and J. R. Steel, editors), Cambridge University Press, Cambridge, 2016, pp. 243-256.

[37] J. R. SteEL and W. H. Woodin, HOD as a core model, Ordinal Definability and Recursion Theory, the Cabal Seminar, vol. III (A. S. Kechris, B. Löwe, and J. R. Steel, editors), Cambridge University Press, Cambridge, 2016, pp. 257-345.

[38] N. D. Trang, Generalized Solovay measures, the HOD analysis, and the core model induction, $\mathrm{Ph} . \mathrm{D}$. thesis, University of California at Berkeley, 2013.

[39] - Determinacy in L $(\mathbb{R}, \mu)$. Journal of Mathematical Logic, vol. 14 (2014), no. 1, 1450006.

$[40]-$ Structure theory of $L(\mathbb{R}, \mu)$ and its applications, this JourNaL, vol. 80 (2015), no. 1, pp. $29-55$.

[41] S. Uhlenbrock, NOW MÜLler, Pure and hybrid mice with finitely many Woodin cardinals from levels of determinacy, Ph.D. thesis, WWU Münster, 2016.

[42] W.H. Woodin, The Axiom of Determinacy, Forcing Axioms, and the Nonstationary Ideal, De Gruyter Series in Logic and its Applications, De Gruyter, Berlin, 2010.

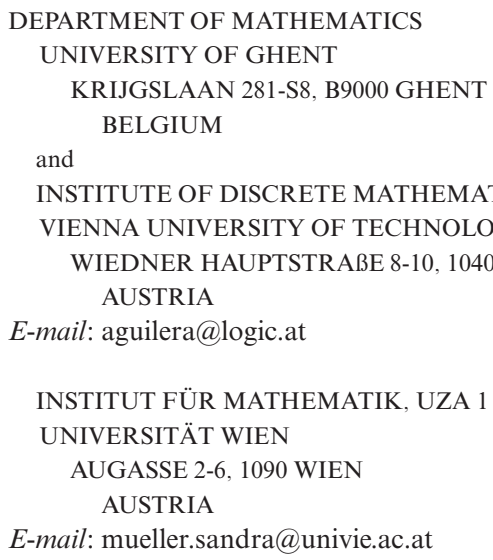

